\title{
Veneziano Amplitudes, Spin Chains and String Models
}

Arkady L. Kholodenkd 1

375 H.L.Hunter Laboratories, Clemson University,Clemson, SC 29634-0973, U.S.A.

In a series of recently published papers we reanalyzed the existing treatments of Veneziano and Veneziano-like amplitudes and the models associated with these amplitudes. In this work we demonstrate that the already obtained new partition function for these amplitudes can be exactly mapped into that for the Polychronakos-Frahm (P-F) spin chain model. This observation allows us to recover many of the existing string-theoretic models, including the most recent ones.

Keywords: Polychronakos-Frahm spin chains; q-deformed harmonic oscillator; Stiltjes-Wigert polynomials; ASEP and spin chains; CFT and Liouville models on the lattice; matrix and topological string models.

\footnotetext{
${ }^{1}$ E-mail address: string@clemson.edu
} 


\section{Introduction}

Since time when quantum mechanics (QM) was born (in 1925-1926) two seemingly opposite approaches for description of atomic and subatomic physics were proposed respectively by Heisenberg and Schrödinger. Heisenberg's approach is aimed at providing the affirmative answer to the following question: Is combinatorics of spectra (of observables) provides sufficient information about microscopic system so that dynamics of such a system can be described in terms of known macroscopic concepts? Schrodinger's approach is exactly opposite and is aimed at providing the affirmative answer to the following question: Using some plausible mathematical arguments is it possible to find an equation which under some prescribed restrictions will reproduce the spectra of observables? Although it is widely believed that both approaches are equivalent, already Dirac in his lectures on quantum field theory [1] noticed (without much elaboration) that Schrodinger's description of QM contains a lot of "dead wood" which can be safely disposed altogether. According to Dirac "Heisenberg's picture of QM is good because Heisenberg's equations of motion make sense". To our knowledge, Dirac's comments were completely ignored, perhaps, because he had not provided enough evidence making Heisenberg's description of QM superior to that of Schrodinger's. In recent papers [2,3] we found examples supporting Dirac's claims. From the point of view of combinatorics, there is not much difference in description of QM, quantum field theory and string theory. Therefore, in this paper we choose Heisenberg's point of view on string theory using results of our recent works in which we re analyzed the existing treatments connecting Veneziano (and Veneziano-like) amplitudes with the respective string-theoretic models. As result, we were able to find new tachyon-free models reproducing Veneziano (and Veneziano-like) amplitudes. In this work results of our papers [4-7] which will be respectively called as Part I, Part II, Part III and Part IV are further developed to bring them in correspondence with those developed by other authors. Without any changes in the already existing formalism, we were able to connect our results with an impressive number of string-theoretic models.

As is well known, all information in high energy physics is obtainable through proper interpretation of the scattering data. It is believed that for sufficiently high energies such data are well described by the phenomenological Regge theory and can be conveniently summarized with help of Chew-Frauthchi plots relating masses to spins (angular momenta), e.g. see book by Collins[8]. Veneziano amplitudes are by design Regge-behaving. Both Regge theory and Veneziano amplitudes emerged before major developments in QCD took place in 70ies. Once these developments took place, naturally, it was of interest to recover the Regge theory from QCD. Even though there are many ways for doing so, to our knowledge, the problem is still not solved completely. This is so for the 
following reasons.

Although the amount of data obtained by perturbative treatments of QCD is quite impressive, e.g. read Ref.[9], these results are not helpful for establishing the Regge-type behavior of QCD. Since such a behavior can be easily established with help of variety of string models, the task lies is connecting these models with QCD. Evidently, such a task is also equally applicable to models reproduced in this work.

The connections between either QCD and spin chains or between strings and spin chains were already discussed in literature for quite some time. Recent paper by Dorey [10] contains may references listing these earlier results. Subsequently, they had been replaced by those whose methods are based on ADS/CFT correspondence. From the point of view of this correspondence, the connections between strings and QCD also can be made through spin chains as it is demonstrated in the seminal paper by Gubser, Klebanov and Polyakov, Ref.[11]. Their ideas were developed in great detail in the paper by Minahan and Zarembo, Ref.[12]. The spectrum of anomalous dimensions of operators in the $\mathcal{N}=4, \mathrm{~N} \rightarrow \infty$ supersymmetric Yang-Mills (Y-M) model (described in terms of the excitation spectrum of the spin chain model) is related to the string spectrum describing hadron masses. To connect these facts with developments in this paper we mention papers by Kruczenski [13], and Cotrone et al.[14]. In both papers spin 1/2 XXX Heisenberg chain was used for description of the excitation spectrum. Furthermore, in the paper by Cotrone et al the explicit connection with the hadron mass spectrum was made. Both papers use ADS/CFT correspondence in their derivations. In this work, we reobtain these spin chain results using combinatorial arguments following Heisenberg's philosophy discussed in some detail in our recent work [2]. In view of this, our derivation is not relying on use of ADS/CFT methods.

It should be noted though that the ADS/CFT is not the only method of connecting QCD with strings. In 1981 't Hooft suggested [15] to reduce the non Abelian QCD to Abelian Ginzburg-Landau (G-L) type theory. The rationale for such an Abelian reduction can be traced back to the work by Nambu [16]. In his work Nambu superimposed G-L theory with the theory of Dirac monopoles to demonstrate quark confinement for mesons. Incidentally, Veneziano amplitudes are suited the most for describing meson resonances, e.g. see Ref.[8]. These are made of just two quarks: quark and antiquark. Thus, if the existence of Abelian reduction would be considered as proven, this then would be equivalent to the proof of quark confinement. Recent numerical studies have provided convincing evidence supporting the idea of quark confinement through monopole condensation, e.g. see Refs. $[17,18]$. Since publication of 't Hooft's paper many theoretical advancements were made, most notably by Cho, Ref.s[19,20], and Kondo, Ref.s [21-23], whose work was motivated by that by Faddeev and his group. Results of this group are summarized in the recent review by Faddeev, Ref.[24]. From this reference it follows that most of efforts to date were spent on description of the massless version of QCD. The excitation spectrum of solitonic knotted-like structures (admitting interpretation in terms of closed strings) provides the spectrum of glueball masses as demonstrated in [22]. In the recent 
paper [25] Auckly, Kapitanski and Speight demonstrated how Skyrme model can be obtained from Faddeev model. Since Skyrme model was used for a long time for description of the baryon spectra, e.g. read Ref.[26], and since already Nambu recognized usefulness of the Abelian reduction for description of the meson spectra, it follows that the Abelian reduction method is also capable of providing sufficient information about QCD in the strong coupling regime.

Since our derivation is not relying on use of ADS/CFT methods, naturally, the question arises: Can Heisenberg-style analysis of this work be useful in deciding which of the methods: ADS/CFT or Abelian reduction is more likely realized in Nature? In our companion paper [27] we provide evidence of superiority of the Abelian reduction over ADS/CFT methods. To avoid duplications, in this work we only develop connections between Veneziano amplitudes, spin chains and various string models without making attempts to relate explicitly these models with QCD.

The rest of this paper is organized as follows. In Sections 2-3 we provide needed physical motivations and background. For this purpose, in Section 2 we replaced mathematically sophisticated derivation of Veneziano partition function developed in [4-6] by a considerably simpler combinatorial derivation of this function analogous to that discussed in [7]. As a by-product of this effort, we were able to uncover connections with spin chains already at this stage of our investigation. To strengthen this connection, in Section 3 we demonstrate that the obtained Veneziano partition function coincides with that for the Polychronakos-Frahm (P-F) partition function for the ferromagnetic spin chain model [28]. In the limit of infinitely long chains such a model coincides with spin 1/2 XXX Heisenberg spin chain. This is explained in Section 4. In the same section we discuss different paths aimed at establishing links between the P-F spin chain and variety of string-theoretic models, including the most recent ones. This is achieved by mapping the combinatorial and analytical properties of the P-F spin chains into analogous properties of spin chains used for description of the stochastic process known in literature as asymptotic simple exclusion process (ASEP). To make our presentation self-contained, Appendix contains some basic information on ASEP sufficient for understanding the results of the main text. In addition, in the main text, we provide some information on the Kardar-Parisi-Zhang (KPZ) and Edwards-Wilkinson (EW) equations which are just different well defined macroscopic limits of the microscopic ASEP equations. This is done with purpose of reproducing a variety of already known stringtheoretic models, including the most recent ones, and to relate them to each other. Section 5 contains some discussion connecting the obtained results with that presented in our companion paper [27]. 


\section{Combinatorics of Veneziano amplitudes and spin chains: qualitative considerations}

In Part I, we noticed that the Veneziano condition for the 4-particle amplitude given by

$$
\alpha(s)+\alpha(t)+\alpha(u)=-1,
$$

where $\alpha(s), \alpha(t), \alpha(u) \in \mathbf{Z}$, can be rewritten in more mathematically suggestive form. To this purpose, following [29], we need to consider additional homogenous equation of the type

$$
\alpha(s) m+\alpha(t) n+\alpha(u) l+k \cdot 1=0
$$

with $m, n, l, k$ being some integers. By adding this equation to (1) we obtain

$$
\alpha(s) \tilde{m}+\alpha(t) \tilde{n}+\alpha(u) \tilde{l}=\tilde{k}
$$

or, equivalently,

$$
n_{1}+n_{2}+n_{3}=\hat{N}
$$

where all entries by design are nonnegative integers. For the multiparticle case this equation should be replaced by

$$
n_{0}+\cdots+n_{k}=N
$$

so that combinatorially the task lies in finding all nonnegative integer combinations of $n_{0}, \ldots, n_{k}$ producing (4). It should be noted that such a task makes sense as long as $N$ is assigned. But the actual value of $N$ is not fixed and, hence, can be chosen quite arbitrarily. Eq.(1) is a simple statement about the energy -momentum conservation. Although the numerical entries in this equation can be changed as we just explained, the actual physical values can be subsequently re obtained by the appropriate coordinate shift. The arbitrariness in selecting $N$ reflects a kind of gauge freedom. As in other gauge theories, we may try to fix the gauge by using some physical considerations. These include, for example, an observation made in Part I that the 4 particle amplitude is zero if any two entries into (1) are the same. This fact prompts us to arrange the entries in (3b) in accordance with their magnitude, i.e. $n_{1} \geq n_{2} \geq n_{3}$. More generally, we can write: $n_{0} \geq n_{1} \geq \cdots \geq n_{k} \geq 12$.

Provided that (4) holds, we shall call such a sequence a partition and shall denote it as $n \equiv\left(n_{0}, \ldots, n_{k}\right)$. If $n$ is a partition of $N$, then we shall write $n \vdash N$. It is well known $[30,31]$ that there is one- to -one correspondence between the Young diagrams and partitions. We would like to use this fact in order to design a partition function capable of reproducing Veneziano (and Venezianolike) amplitudes. Clearly, such a partition function should also make physical sense. Hence, we would like to provide some qualitative arguments aimed at

\footnotetext{
${ }^{2}$ The last inequality: $n_{k} \geq 1$, is chosen only for the sake of comparison with the existing literature conventions, e.g. see Ref.[30].
} 
convincing our readers that such a partition function does exist and is physically sensible.

We begin with observation that there is one- to- one correspondence between the Young tableaux and directed random walk: 3 . It is useful to recall details of this correspondence now. To this purpose we need to consider a square lattice and to place on it the Young diagram associated with some particular partition.

Let us choose some $\tilde{n} \times \tilde{m}$ rectangle 4 so that the Young diagram occupies the left part of this rectangle. We choose the upper left vertex of the rectangle as the origin of the $x y$ coordinate system whose $y$ axis (South direction) is directed downwards and $x$ axis is directed Eastwards. Then, the South-East boundary of the Young diagram can be interpreted as directed (that is without self intersections) random walk which begins at $(0,-\tilde{m})$ and ends at $(\tilde{n}, 0)$. Evidently, such a walk completely determines the diagram. The walk can be described by a sequence of 0 's and 1's. Say, 0 for the $x-$ step move and 1 for the $y$ - step move. The totality $\mathcal{N}$ of Young diagrams which can be placed into such a rectangle is in one-to-one correspondence with the number of arrangements of 0's and 1's whose total number is $\tilde{m}+\tilde{n}$. Recalling the Fermi statistics, the number $\mathcal{N}$ can be easily calculated and is given by $\mathcal{N}=(m+n) ! / m ! n ! 5$. It can be represented in two equivalent ways

$$
\begin{aligned}
(m+n) ! / m ! n ! & =\frac{(n+1)(n+2) \cdots(n+m)}{m !} \equiv\left(\begin{array}{c}
n+m \\
m
\end{array}\right) \\
& =\frac{(m+1)(m+2) \cdots(n+m)}{n !} \equiv\left(\begin{array}{c}
m+n \\
n
\end{array}\right) .
\end{aligned}
$$

Let now $p(N ; k, m)$ be the number of partitions of $N$ into $\leq k$ nonnegative parts, each not larger than $m$. Consider the generating function of the following type

$$
\mathcal{F}(k, m \mid q)=\sum_{N=0}^{S} p(N ; k, m) q^{N}
$$

where the upper limit $S$ will be determined shortly below. It is shown in Refs. [30,31] that $\mathcal{F}(k, m \mid q)=\left[\begin{array}{c}k+m \\ m\end{array}\right]_{q} \equiv\left[\begin{array}{c}k+m \\ k\end{array}\right]_{q}$ where, for instance, $\left[\begin{array}{c}k+m \\ m\end{array}\right]_{q=1}=$ $\left(\begin{array}{c}k+m \\ m\end{array}\right) 6$. From this result it should be clear that the expression $\left[\begin{array}{c}k+m \\ m\end{array}\right]_{q}$

\footnotetext{
${ }^{3}$ Furthermore, it is possible to map bijectively such type of random walk back into Young diagram with only two rows, e.g. read [32], page 5. This allows us to make a connection with spin chains at once. In this work we are not going to use this route to spin chains in view of the simplicity of alternative approaches discussed in this section.

${ }^{4}$ Parameters $\tilde{n}$ and $\tilde{m}$ will be specified shortly below.

${ }^{5}$ We have suppressed the tildas for $n$ and $m$ in this expression since these parameters are going to be redefined below anyway.

${ }^{6}$ On page 15 of the book by Stanley [31], one can find that the number of solutions $N(n, k)$ in positive integers to $y_{1}+\ldots+y_{k}=n+k$ is given by $\left(\begin{array}{c}n+k-1 \\ k-1\end{array}\right)$ while the number of
} 
is the $q$-analog of the binomial coefficient $\left(\begin{array}{c}k+m \\ m\end{array}\right)$. In literature [30,31] this $q-$ analog is known as the Gaussian coefficient. Explicitly, it is defined as

$$
\left[\begin{array}{l}
a \\
b
\end{array}\right]_{q}=\frac{\left(q^{a}-1\right)\left(q^{a-1}-1\right) \cdots\left(q^{a-b+1}-1\right)}{\left(q^{b}-1\right)\left(q^{b-1}-1\right) \cdots(q-1)}
$$

for some nonegative integers $a$ and $b$. From this definition we anticipate that the sum defining generating function $\mathcal{F}(k, m \mid q)$ in (6) should have only finite number of terms. Eq.(7) allows easy determination of the upper limit $S$ in the sum (6). It is given by $k m$. This is just the area of the $k \times m$ rectangle. In view of the definition of $p(N ; k, m)$, the number $m=N-k$. Using this fact (6) can be rewritten as: $\mathcal{F}(N, k \mid q)=\left[\begin{array}{c}N \\ k\end{array}\right]_{q}$. This expression happens to be the Poincare' polynomial for the Grassmannian $\operatorname{Gr}(m, k)$ of the complex vector space $\mathbf{C}^{N}$ of dimension $N$ as can be seen from page 292 of the book by Bott and $\mathrm{Tu},[33]^{7}$. From this (topological) point of view the numerical coefficients, i.e. $p(N ; k, m)$, in the $q$ expansion of $(6)$ should be interpreted as Betti numbers of this Grassmannian. They can be determined recursively using the following property of the Gaussian coefficients [31], page 26,

$$
\left[\begin{array}{l}
n+1 \\
k+1
\end{array}\right]_{q}=\left[\begin{array}{c}
n \\
k+1
\end{array}\right]_{q}+q^{n-k}\left[\begin{array}{l}
n \\
k
\end{array}\right]_{q}
$$

and taking into account that $\left[\begin{array}{l}n \\ 0\end{array}\right]_{q}=1$. We refer our readers to Part II for mathematical proof that $\mathcal{F}(N, k \mid q)$ is indeed the Poincare' polynomial for the complex Grassmannian. With this fact proven, we notice that, due to relation $m=N-k$, it is sometimes more convenient for us to use the parameters $m$ and $k$ rather than $N$ and $k$. With such a replacement we obtain:

$$
\begin{aligned}
\mathcal{F}(k, m & \mid q)=\left[\begin{array}{c}
k+m \\
k
\end{array}\right]_{q}=\frac{\left(q^{k+m}-1\right)\left(q^{k+m-1}-1\right) \cdots\left(q^{m+1}-1\right)}{\left(q^{k}-1\right)\left(q^{k-1}-1\right) \cdots(q-1)} \\
& =\prod_{i=1}^{k} \frac{1-q^{m+i}}{1-q^{i}} .
\end{aligned}
$$

This result is of central importance for this work. In Part II a considerably more sophisticated mathematical apparatus was used to obtain it (e.g. see equation (6.10) of this reference and arguments leading to it).

In the limit $q \rightarrow 1$ Eq.(9) reduces to $\mathcal{N}$ as required. To make connections with results known in physics literature we need to re scale $q^{\prime} s$ in (9), e.g. let

solutions in nonnegative integers to $x_{1}+\ldots+x_{k}=n$ is $\left(\begin{array}{c}n+k \\ k\end{array}\right)$. Careful reading of Page 15 indicates however that the last number refers to solution in nonnegative integers of the equation $x_{0}+\ldots+x_{k}=n$. This fact was used essentially in (1.21) of Part I.

${ }^{7}$ To make a comparison it is sufficient to replace parameters $t^{2}$ and $n$ in Bott and Tu book by $q$ and $N$. 
$q=t^{\frac{1}{2}}$. Substitution of such an expression back into (9) and taking the limit $t \rightarrow 1$ again produces $\mathcal{N}$ in view of (5). This time, however, we can accomplish more. By noticing that in (4) the actual value of $N$ deliberately is not yet fixed and taking into account that $m=N-k$ we can fix $N$ by fixing $m$. Specifically, we would like to choose $m=1 \cdot 2 \cdot 3 \cdots k$ and with such a choice we would like to consider a particular term in the product (9), e.g.

$$
S(i)=\frac{1-t^{1+\frac{m}{i}}}{1-t} .
$$

In view of our "gauge fixing" the ratio $m / i$ is a positive integer by design. This means that we are having a geometric progression. Indeed, if we rescale $t$ again $: t \rightarrow t^{2}$, we then obtain:

$$
S(i)=1+t^{2}+\cdots+t^{2 \hat{m}}
$$

with $\hat{m}=\frac{m}{i}$. Written in such a form the above sum is just the Poincare' polynomial for the complex projective space $\mathbf{C} \mathbf{P}^{\hat{m}}$. This can be seen by comparing pages 177 and 269 of the book by Bott and Tu [33]. Hence, at least for some $m$ 's, the Poincare' polynomial for the Grassmannian in just the product of the Poincare' polynomials for the complex projective spaces of known dimensionalities. For $m$ just chosen, in the limit $t \rightarrow 1$, we reobtain back the number $\mathcal{N}$ as required. This physically motivating process of gauge fixing just described can be replaced by more rigorous mathematical arguments. The recursion relation (8) indicates that this is possible. The mathematical details leading to factorization which we just described can be found, for instance, in the Ch-3 of lecture notes by Schwartz [34]. The relevant physics emerges by noticing that the partition function $Z(J)$ for the particle with spin $J$ is given by [35]

$$
\begin{aligned}
Z(J) & =\operatorname{tr}\left(e^{-\beta H(\sigma)}\right)=e^{c J}+e^{c(J-1)}+\cdots+e^{-c J} \\
& =e^{c J}\left(1+e^{-c}+e^{-2 c}+\cdots+e^{-2 c J}\right),
\end{aligned}
$$

where $c$ is known constant. Evidently, up to a constant, $Z(J) \simeq S(i)$. Since mathematically the result (12) is the Weyl character formula, this observation brings the classical group theory into our discussion. More importantly, because the partition function for the particle with spin $J$ can be written in the language of $\mathrm{N}=2$ supersymmetric quantum mechanical mode 8 , as demonstrated by Stone [35] and others [36], the connection between the supersymmetry and the classical group theory is evident. It was developed in Parts III and IV.

In view of arguments presented above, the Poincare' polynomial for the Grassmannian can be interpreted as a partition function for some kind of a spin chain made of apparently independent spins of various magnitudes. These qualitative arguments we would like to make more mathematically and physically rigorous. The first step towards this goal is made in the next section.

\footnotetext{
${ }^{8}$ We hope that no confusion is made about the meaning of $\mathrm{N}$ in the present case.

${ }^{9}$ In such a context it can be vaguely considered as a variation on the theme of the Polyakov rigid string (Grassmann $\sigma$ model, Ref.[37], pages 283-287), except that now it is exactly solvable in the qualitative context just described and, below, in the mathematically rigorous context.
} 


\section{Connection with the Polychronakos-Frahm spin chain model}

The Polychronakos-Frahm (P-F) spin chain model was originally proposed by Polychronakos and described in detail in [28]. Frahm [38] motivated by the results of Polychronakos made additional progress in elucidating the spectrum and thermodynamic properties of this model so that it had become known as the P-F model. Subsequently, many other researchers have contributed to our understanding of this exactly integrable spin chain model. Since this paper is not a review, we shall quote only works on P-F model which are of immediate relevance.

Following [28], we begin with some description of the P-F model. Let $\sigma_{i}^{a}(a=$ $\left.1,2, \ldots, n^{2}-1\right)$ be $S U(n)$ spin operator of i-th particle and let the operator $\sigma_{i j}$ be responsible for a spin exchange between particles $i$ and $j$, i.e.

$$
\sigma_{i j}=\frac{1}{n}+\sum_{a} \sigma_{i}^{a} \sigma_{j}^{a}
$$

In terms of these definitions, the Calogero-type model Hamiltonian can be written as $[39,40]$

$$
\mathcal{H}=\frac{1}{2} \sum_{i}\left(p_{i}^{2}+\omega^{2} x_{i}^{2}\right)+\sum_{i<j} \frac{l\left(l-\sigma_{i j}\right)}{\left(x_{i}-x_{j}\right)^{2}}
$$

where $l$ is some parameter. The P-F model is obtained from the above model in the limit $l \rightarrow \pm \infty$. Upon proper rescaling of $\mathcal{H}$ in (14), in this limit one obtains

$$
\mathcal{H}_{\mathcal{P}-\mathcal{F}}=-\operatorname{sign}(l) \sum_{i<j} \frac{\sigma_{i j}}{\left(x_{i}-x_{j}\right)^{2}}
$$

where the coordinate $x_{i}$ minimizes the potential for the rescaled Calogero mode10, that is

$$
\omega^{2} x_{i}=\sum_{i<j} \frac{2}{\left(x_{i}-x_{j}\right)^{3}} .
$$

It should be noted that $\mathcal{H}_{\mathcal{P}-\mathcal{F}}$ is well defined without such a minimization, that is for arbitrary real parameters $x_{i}$. This fact is explained in detail in our companion paper[27]. In the large $l$ limit the spectrum of $\mathcal{H}$ is decomposable as

$$
E=E_{\mathcal{C}}+l E_{\mathcal{P}-\mathcal{F}},
$$

where $E_{C}$ is the spectrum of the spinless Calogero model while $E_{\mathcal{P}-\mathcal{F}}$ is the spectrum of the P-F model. In view of such a decomposition, the partition function for the Hamiltonian $\mathcal{H}$ at temperature $T$ can be written as a product: $\mathrm{Z}_{\mathcal{H}}(T)=\mathrm{Z}_{\mathcal{C}}(T) \mathrm{Z}_{\mathcal{P}-\mathcal{F}}(T / l)$. From here, one formally obtains the result:

$$
Z_{\mathcal{P}-\mathcal{F}}(T)=\lim _{l \rightarrow \infty} \frac{Z_{\mathcal{H}}(l T)}{Z_{\mathcal{C}}(T)} .
$$

\footnotetext{
${ }^{10}$ The Calogero model is obtainable from the Hamiltonian (14) if one replaces the spin exchange operator $\sigma_{i j}$ by 1 . Since we are interested in the large $l$ limit, one can replace the factor $l(l-1)$ by $l^{2}$ in the interaction term.
} 
It implies that the spectrum of the P-F spin chain can be obtained if both the total and the Calogero partition functions can be calculated. In [28] Polychronakos argued that $Z_{\mathcal{C}}(T)$ is essentially a partition function of $N$ noninteracting harmonic oscillators. Thus, we obtain

$$
Z_{\mathcal{C}}(N ; T)=\prod_{i=1}^{N} \frac{1}{1-q^{i}}, q=\exp (-\beta), \beta=\left(k_{B} T\right)^{-1} .
$$

Furthermore, the partition function $Z_{\mathcal{H}}(T)$ according to Polychronakos can be obtained using $Z_{\mathcal{C}}(N ; T)$ as follows. Consider the grand partition function of the type

$$
\Xi=\sum_{N=0}^{\infty} Z_{n}(N ; T) y^{N} \equiv\left(\sum_{L=0}^{\infty} Z_{\mathcal{C}}(L ; T) y^{L}\right)^{n}
$$

where $n$ is the number of flavor:11. Using this definition we obtain

$$
Z_{n}(N ; T)=\sum_{\Sigma_{i} k_{i}=N} \prod_{i=1}^{n} Z_{\mathcal{C}}\left(k_{i} ; T\right) .
$$

Next, Polychronakos identifies $Z_{n}(N ; T)$ with $Z_{\mathcal{H}}(T)$. Then, with help of (18) the partition function $Z_{\mathcal{P}-\mathcal{F}}(T)$ is obtained straightforwardly as

$$
Z_{\mathcal{P}-\mathcal{F}}(N ; T)=\sum_{\Sigma_{i} k_{i}=N} \frac{\prod_{i=1}^{N}\left(1-q^{i}\right)}{\prod_{i=1}^{n} \prod_{r=1}^{k_{i}}\left(1-q^{r}\right)}
$$

Consider this result for a special case: $n=2$. It is convenient to evaluate the ratio first before calculating the sum. Thus, we obtain

$$
\frac{\prod_{i=1}^{N}\left(1-q^{i}\right)}{\prod_{i=1}^{2} \prod_{r=1}^{k_{i}}\left(1-q^{r}\right)}=\frac{(1-q) \cdots\left(1-q^{N}\right)}{(1-q) \cdots\left(1-q^{k}\right)(1-q) \cdots\left(1-q^{N-k}\right)} \equiv \mathcal{F}(N, k \mid q),
$$

where the Poincare' polynomial $\mathcal{F}(N, k \mid q)$ for the Grassmanian of the complex vector space $\mathbf{C}^{N}$ of dimension $N$ was obtained in the previous section. Indeed (23) can be trivially brought into the same form as that given in our Eq.(9) using the relation $m+k=N$. To bring Eq.(9) in correspondence with equation (4.1) of Polychronakos [28], we use the second equality given in Eq.(9) in which we make a substitution: $m=N-k$. After this replacement, Eq.(22) acquires the form

$$
Z_{\mathcal{P}-\mathcal{F}}^{f}(N ; T)=\sum_{k=0}^{N} \prod_{i=0}^{k} \frac{1-q^{N-i+1}}{1-q^{i}}
$$

\footnotetext{
${ }^{11}$ That is $n$ the same number as $n$ in $S U(n)$.
} 
coinciding with Eq.(4.1) by Polychronakos. This equation corresponds to the ferromagnetic version of the P-F spin chain model. To obtain the antiferromagnetic version of the model requires us only to replace $q$ by $q^{-1}$ in (24) and to multiply the whole r.h.s. by some known power of $q$. Since this factor will not affect thermodynamics, following Frahm [38], we shall ignore it. As result, we obtain

$$
Z_{\mathcal{P}-\mathcal{F}}^{a f}(N ; T)=\sum_{k=0}^{N} q^{(N / 2-k)^{2}} \prod_{i=0}^{k} \frac{1-q^{N-i+1}}{1-q^{i}},
$$

in accord with Frahm's Eq.(21). This result is analyzed further in the next section playing the central role for this paper.

\section{Connections with WZNW model and XXX $\mathrm{s}=1 / 2$ Heisenberg antiferromagnetic spin chain}

\subsection{General remarks}

To establish these connections we follow work by Hikami [41]. For this purpose, we introduce the notation

$$
(q)_{n}=\prod_{i=1}^{n}\left(1-q^{i}\right)
$$

allowing us to rewrite (25) in the equivalent form

$$
Z_{\mathcal{P}-\mathcal{F}}^{a f}(N ; T)=\sum_{k=0}^{N} q^{(N / 2-k)^{2}} \prod_{i=0}^{k} \frac{1-q^{N-i+1}}{1-q^{i}}=\sum_{k=0}^{N} q^{(N / 2-k)^{2}} \frac{(q)_{N}}{(q)_{k}(q)_{N-k}}
$$

Consider now the limiting case $(N \rightarrow \infty)$ of the obtained expression. For this purpose we need to take into account that

$$
\lim _{N \rightarrow \infty}\left[\begin{array}{c}
N \\
k
\end{array}\right]_{q}=\frac{1}{(q)_{k}} .
$$

To use this asymptotic result in (27) it is convenient to consider separately the cases of $N$ being even and odd. For instance, if $N$ is even, we can write: $N=2 m$. In such a case we can introduce new summation variables: $l=k-m$ and/or $l=m-k$. Then, in the limit $N \rightarrow \infty$ (that is $m \rightarrow \infty$ ) we obtain asymptotically

$$
Z_{\mathcal{P}-\mathcal{F}}^{a f}(\infty ; T)=\frac{1}{(q)_{\infty}} \sum_{i=-\infty}^{\infty} q^{i^{2}} .
$$

in accord with [41]. Analogously, if $N=2 m+1$, we obtain instead

$$
Z_{\mathcal{P}-\mathcal{F}}^{a f}(\infty ; T)=\frac{1}{(q)_{\infty}} \sum_{i=-\infty}^{\infty} q^{\left(i+\frac{1}{2}\right)^{2}}
$$


According to Melzer [42] and Kedem, McCoy and Melzer [43], the obtained partition functions coincide with the Virasoro characters for $\mathrm{SU}_{1}(2)$ WZNW model describing the conformal limit of the XXX $(\mathrm{s}=1 / 2)$ antiferromagnetic spin chain [44]. Even though equations (29a) and (29b) provide the final result, they do not reveal their physical content. This task was accomplished in part in the same papers where connection with the excitation spectrum of the XXX antiferromagnetic chain was made. To avoid repetitions, below we arrive at these conclusions using different arguments. By doing so many new and unexpected connections with other stochastic models will be uncovered.

\subsection{Method of generating functions and q-deformed har- monic oscillator}

We begin with definitions. In view of equations (9),(24) and (27), we would like to introduce the Galois number $G_{N}$ via

$$
G_{N}=\sum_{k=0}^{N}\left[\begin{array}{c}
N \\
k
\end{array}\right]_{q}
$$

This number can be calculated recursively as it was shown by Goldman and Rota [45] with the result

$$
G_{N+1}=2 G_{N}+\left(q^{N}-1\right) G_{N-1} .
$$

Alternative proof was given by Kac and Cheung [46]. To calculate $G_{N}$ we have to take into account that $G_{0}=1$ and $G_{1}=2$. These results can be used as a reference when one attempts to calculate the related Rogers-Szego (R-S) polynomial $H_{N}(t)$ defined as [47]

$$
H_{N}(t ; q):=\sum_{k=0}^{N}\left[\begin{array}{c}
N \\
k
\end{array}\right]_{q} t^{k}
$$

so that $H_{N}(1)=G_{N} 12$. Using Ref.[46] once again, we find that $H_{N}(t)$ obeys the following recursion relation

$$
H_{N+1}(t)=(1+t) H_{N}(t)+\left(q^{N}-1\right) t H_{N-1}(t)
$$

which for $t=1$ coincides with (31) as required. The above recursion relation is supplemented with initial conditions. These are : $H_{0}=1$ and $H_{1}=1+t$.

At this point we would like to remind our readers that for $t=1$ according to $(24),(30)$ and (32) we obtain: $Z_{\mathcal{P}-\mathcal{F}}^{f}(N ; T)=G_{N}$. Hence, by calculating $H_{N}(t)$ we also shall obtain the partition function for the P-F chain.

To proceed with such calculations, we follow Ref.[48]. In particular, we consider first the auxiliary recursion relation for Hermite polynomials:

$$
H_{n+1}(x)=2 x H_{n}(x)-2 n H_{n-1}(x)
$$

\footnotetext{
${ }^{12}$ For brevity, unless needed explicitly, we shall suppress the argument $q$ in $H_{N}(t ; q)$.
} 
supplemented by the differential relation

$$
\frac{d}{d x} H_{n}(x)=2 n H_{n-1}(x)
$$

which, in view of (34a), can be conveniently rewritten as

$$
H_{n+1}(x)=\left(2 x-\frac{d}{d x}\right) H_{n}(x) .
$$

This observation prompts us to introduce the raising operator $R=2 x-\frac{d}{d x}$ so that we obtain:

$$
R^{n} H_{0}(x)=H_{n}(x) .
$$

The lowering operator can be now easily obtained again using (34). Indeed, we obtain

$$
\frac{1}{2} \frac{d}{d x} H_{n}(x) \equiv L H_{n}(x)=n H_{n-1}(x)
$$

so that $[L, R]=1$ as required. Based on this, the number operator $N$ can be obtained as $N=R L$ so that $N H_{n}(x)=n H_{n}(x)$ or, explicitly, using provided definitions, we obtain:

$$
\left(\frac{d^{2}}{d x^{2}}-2 x \frac{d}{d x}+2 n\right) H_{n}(x)=0 .
$$

Evidently, we can write: $R|n>=| n+1>, L|n>=| n-1>$ and , $<m \mid n>=n ! \delta_{m n}$ as usual.

We would like now to transfer all these results to our main object of interestthe recursion relation (33). To this purpose, we introduce the difference operator $\Delta$ via

$$
\Delta H_{N}(t):=H_{N}(t)-H_{N}(q t) .
$$

Using (32) we obtain now

$$
\Delta H_{N}(t)=\left(1-q^{N}\right) t H_{N-1}(t),
$$

where we took into account that

$$
\left[\begin{array}{c}
N \\
k
\end{array}\right]_{q}=\left[\begin{array}{c}
N \\
N-k
\end{array}\right]_{q}
$$

Using this result in (33) we obtain at once

$$
H_{N+1}(t)=[(1+t)-\Delta] H_{N}(t) .
$$

This, again, can be looked upon as a definition of a raising operator so that we can formally rewrite (41) as

$$
\mathcal{R} H_{N}(t)=H_{N+1}(t) .
$$


The lowering operator can be defined now as

$$
\mathcal{L}:=\frac{1}{x} \Delta
$$

so that

$$
\mathcal{L} H_{N}(t)=\left(1-q^{N}\right) H_{N-1}(t) .
$$

The action of the number operator $\mathcal{N}=\mathcal{R} \mathcal{L}$ is now straightforward, i.e.

$$
\mathcal{N} H_{N}(t)=\left(1-q^{N}\right) H_{N}(t) .
$$

Following Kac and Cheung $[\mathbf{3 2}]$ we introduce the $q$-derivative via

$$
D_{q} f(x):=\frac{f(q x)-f(x)}{x(q-1)} .
$$

By combining this result with (38) we obtain,

$$
D_{q} f(x)=\frac{\Delta f(x)}{x(1-q)} .
$$

This allows us to rewrite the raising and lowering operators in terms of $q$-derivatives. Specifically, we obtain

$$
\tilde{\mathcal{R}}:=(1+t)-(1-q) t D_{q},
$$

and

$$
\tilde{\mathcal{L}}:=D_{q} .
$$

While for the raising operator rewritten in such a way Eq.(42) still holds, for the lowering operator $\tilde{\mathcal{L}}$ we now obtain:

$$
\tilde{\mathcal{L}} H_{N}(t)=\frac{1-q^{N}}{1-q} H_{N-1}(t) \equiv[N] H_{N-1}(t) .
$$

The number operator $\mathcal{N}_{q}$ is acting in this case as

$$
\mathcal{N}_{q} H_{N}(t)=[N] H_{N}(t) .
$$

We would like to connect these results with those available in literature on $q$-deformed harmonic oscillator. Following Chaichan et al [49], we notice that the undeformed oscillator algebra is given in terms of the following commutation relations

$$
\begin{gathered}
a a^{+}-a^{+} a=1 \\
{[N, a]=-a}
\end{gathered}
$$

and

$$
\left[N, a^{+}\right]=a^{+} .
$$

In these relations it is not assumed a priori that $N=a^{+} a$ and, therefore, this algebra is formally different from the traditionally used $\left[a, a^{+}\right]=1$ for 
the harmonic oscillator. This observation allows us to introduce the central element $Z=N-a^{+} a$ which is zero for the standard oscillator algebra. The deformed oscillator algebra can be obtained now using Eq.s(52) in which one should replace $(52 \mathrm{a})$ by

$$
a a^{+}-q a^{+} a=1 \text {. }
$$

as it is done in [50]. Consider now the combination $K:=\mathcal{L} \mathcal{R}-q \mathcal{N}$ acting on $H_{N}$ using previously introduced definitions. Simple calculation produces an operator identity: $\mathcal{L} \mathcal{R}-q \mathcal{N}=1$, so that we can formally make a provisional identification $: \mathcal{L} \rightarrow a$ and $\mathcal{R} \rightarrow a^{+}$. To proceed, we need to demonstrate that with such an identification equations ( $52 \mathrm{~b}, \mathrm{c}$ ) hold as well. For this to happen, we should properly normalize our wave function in accord with known procedure for the harmonic oscillator where we have to use $\left|n>=\frac{1}{\sqrt{n !}}\left(a^{+}\right)^{n}\right| 0>$. In the present case, we have to use $\left|N>=\frac{1}{\sqrt{[N] !}}(\mathcal{R})^{n}\right| 0>$ as the basis wavefunction while making an identification: $\mid 0>=H_{0}(t)$. The eigenvalue equation (51), when written explicitly, acquires the following form:

$$
\left[t D_{q}^{2}-\frac{1+t}{1-q} D_{q}+\frac{[N]}{1-q}\right] H_{N}(t)=0
$$

\subsection{The limit $q \rightarrow 1^{ \pm}$and emergence of the Stieltjes-Wigert polynomials}

Obtained results need further refinements for the following reasons. Although the recursion relations (33), (34) look similar, in the limit $q \rightarrow 1^{ \pm}$equation (33) is not transformed into (34). Accordingly, (53) is not converted into equation for Hermite polynomials known for harmonic oscillator. Fortunately, the situation can be repaired in view of recent paper by Karabulut [51] who spotted and corrected some error in the influential earlier paper by Macfarlane [52]. Following the logic of these papers, we define the translation operator $T(s)$ as $T(s):=e^{s \frac{\partial}{\partial x}}$. Using this definition, the creation $a^{\dagger}$ and annihilation $a$ operators are defined as follows

$$
a^{\dagger}=\frac{1}{\sqrt{1-q}}\left[q^{x+\frac{1}{4}}-T^{\frac{-1}{2}}(s)\right] T^{\frac{-1}{2}}(s)
$$

where $T^{\frac{1}{2}}(s)=e^{\frac{s}{2} \frac{\partial}{\partial x}}$ and, accordingly,

$$
a=\frac{1}{\sqrt{1-q}} T^{\frac{1}{2}}(s)\left[q^{x+\frac{1}{4}}-T^{\frac{1}{2}}(s)\right] .
$$

Under such conditions, the inner product is defined in the standard way, that is

$$
(f, g)=\int_{-\infty}^{\infty} f^{*}(x) g(x) d x
$$


so that $\left(q^{x}\right)^{\dagger}=q^{x}$ and $(\partial / \partial x)^{\dagger}=-(\partial / \partial x)$ thus making the operator $a^{\dagger}$ to be a conjugate of $a$ in a usual way. The creation-annihilation operators just defined satisfy commutation relation $(52 \mathrm{~d})$. At the same time, the combination $a^{\dagger} a$ while acting on the wave functions $\Psi_{n}$ (to be defined below) produces equation similar to $(51)$, that is

$$
a^{\dagger} a \Psi_{n}=[n] \Psi_{n} \equiv \lambda_{n} \Psi_{n}
$$

Furthermore, it can be shown, that

$$
a \Psi_{n}=\sqrt{\lambda_{n}} \Psi_{n-1} \text { and } a^{\dagger} \Psi_{n}=\sqrt{\lambda_{n+1}} \Psi_{n+1}
$$

in accord with previously obtained results. Next, we would like to obtain the wave function $\Psi_{n}$ explicitly. To this purpose we start with the ground state $a \Psi_{0}=0$ and use (54b) to get (for $\left.s=1 / 2\right)$ the following result

$$
\Psi_{0}\left(x+\frac{1}{2}\right)=q^{\frac{1}{4}+x} \Psi_{0}(x) .
$$

Let $w(x)$ be some yet unknown function. Then, it is appropriate to look for solution of (58) in the form

$$
\Psi_{0}(x)=\text { const } \cdot w(x) q^{x^{2}},
$$

provided that the function $w(x)$ is periodic: $w(x)=w(x+1 / 2)$. The normalized ground state function acquires then the following look

$$
\Psi_{0}(x)=\alpha_{w} w(x) q^{x^{2}}
$$

where the constant $\alpha_{w}$ is given by

$$
\alpha_{w}=\left(\int_{-\infty}^{\infty} d x\left|q^{x^{2}} w(x)\right|^{2}\right)^{-\frac{1}{2}}
$$

Using this result, $\Psi_{n}$ can be constructed in a standard way through use of the raising operators. There is, however, a faster way to obtain the desired result. To this purpose, in view of $(59 \mathrm{~b})$, suppose that $\Psi_{n}(x)$ can be decomposed as follows

$$
\Psi_{n}(x)=\frac{\alpha_{w} w(x)}{\sqrt{(q, q)_{n}}} \sum_{k=0}^{\infty} C_{k}^{n}(q)(-1)^{k} q^{(n-k) / 2} q^{(x-k)^{2}}
$$

where $(q, q)_{n}=(1-q)\left(1-q^{2}\right) \cdots\left(1-q^{n}\right)$ and $C_{k}^{n}(q)$ is to be determined. To do so, by applying the operator $a^{\dagger} / \sqrt{\lambda_{n+1}}$ to (60) and taking into account that $T^{-\frac{1}{2}} w(x)=w(x-1 / 2)=w(x)$ (in view of the periodicity of $w(x)$ ) we end up with the recursion relation for $C_{k}^{n}(q)$ :

$$
C_{k}^{n+1}(q)=q^{k} C_{k}^{n}(q)+C_{k-1}^{n}(q) .
$$

\footnotetext{
${ }^{13}$ The rationale for choosing $s=1 / 2$ is explained in the same reference.
} 
This relation should be compared with that given by (8). Andrews [47], page 35 , demonstrated that (8) and (61) are equivalent. That is,

$$
C_{k}^{n}(q)=\left[\begin{array}{c}
n \\
k
\end{array}\right]_{q} .
$$

This result implies that, indeed, up to a constant, the obtained wavefunction should be related to the Rogers-Szego polynomial. The obtained relation is nontrivial nevertheless. We would like to discuss this nontriviality in some detail now.

Following [51,53], let $q=e^{-c^{2}}$, where $c$ is some nonegative number. Introduce the distributed Gaussian polynomials via

$$
\Phi_{n}(x)=\sum_{k=0}^{\infty} C_{k}^{n}(q)(-1)^{k} q^{-k / 2} q^{(x-k)^{2}} .
$$

These polynomials satisfy the following orthogonality relation:

$$
\int_{-\infty}^{\infty} \Phi_{n}(x) \Phi_{m}(x) d x=\left\|\Phi_{n}(x)\right\|^{2} \delta_{m n}
$$

with 14

$$
\left\|\Phi_{n}(x)\right\|=\left(\frac{\pi}{2 c^{2}}\right)^{\frac{1}{4}} q^{-\frac{n}{2}} \sqrt{(q, q)_{n}} .
$$

This result calls for a change in normalization of $\Phi_{n}(x)$, i.e., $\phi_{n}(x)=\frac{\Phi_{n}(x)}{\left\|\Phi_{n}(x)\right\|}$. Under such conditions $\phi_{n}(x)$ coincides with $\Psi_{n}(x)$, provided that $w(x)=1$. Introduce new variable $u=q^{-2 x}$, and consider a shift: $\Phi_{n}(x) \rightarrow \Phi_{n}(x-s)$. Using (63), we can write

$$
\Phi_{n}(x-s)=u^{s} \exp \left\{-(\ln u)^{2} /(-4 \ln q)\right\} P_{n}(u ; s),
$$

where

$$
P_{n}(u ; s)=\sum_{k=0}^{\infty} C_{k}^{n}(q)(-1)^{k} q^{-k / 2} q^{(s+k)^{2}} u^{k} .
$$

The orthogonality relation (64) is converted then into

$$
\int_{0}^{\infty} d u u^{2 s-1} \exp \left\{-(\ln u)^{2} /(-4 \ln q)\right\} P_{n}(u ; s) P_{m}(u ; s)=\delta_{m n} .
$$

In view of (67), consider now a special case: $s=1 / 2$. Then, the weight function is known as lognormal distribution and polynomials $P_{n}(u ; 1 / 2)$ (up to a

$$
{ }^{14} \text { Notice that } \alpha=\left(\int_{-\infty}^{\infty} d x q^{2 x^{2}}\right)^{-\frac{1}{2}}=\left(\frac{\pi}{2 c^{2}}\right)^{-\frac{1}{4}}
$$


constant ) are known as Stieltjes-Wigert (S-W) polynomials. Their physical relevance will be discussed below in Subsection 4.6. In the meantime, we introduce the Fourier transform of $f(x)$ in the usual way as

$$
\int_{-\infty}^{\infty} d x \exp (2 \pi i \theta x) f(x)=f(\theta)
$$

Then, the Parseval relation implies:

$$
\int_{-\infty}^{\infty} \Phi_{n}(x) \Phi_{m}(x) d x=\int_{-\infty}^{\infty} \Phi_{n}(\theta) \Phi_{m}(\theta) d x=\left\|\Phi_{n}(x)\right\|^{2} \delta_{m n},
$$

causing

$$
\Phi_{n}(\theta)=\left(\frac{\pi}{c^{2}}\right)^{\frac{1}{4}} \exp \left(-(\pi / c) \theta^{2}\right) \sum_{k=0}^{\infty} C_{k}^{n}(q)\left(-q^{-\frac{1}{2}} e^{2 \pi i \theta}\right)^{k}
$$

Comparison between these results and Eq.(32) produces

$$
\int_{-\infty}^{\infty} H_{n}\left(-q^{-\frac{1}{2}} e^{-2 \pi i \theta} ; q\right) H_{m}\left(-q^{-\frac{1}{2}} e^{-2 \pi i \theta} ; q\right) \exp \left(-2(\pi / c) \theta^{2}\right)=\left(\frac{c}{2 \pi}\right)^{\frac{1}{2}} q^{-n}(q, q)_{n} \delta_{m n}
$$

which can be alternatively rewritten as

$$
\int_{0}^{1} H_{n}\left(-q^{-\frac{1}{2}} e^{-2 \pi i \theta}\right) H_{m}\left(-q^{-\frac{1}{2}} e^{-2 \pi i \theta}\right) \vartheta_{3}(2 \pi \theta ; q) d \theta=q^{-n}(q, q)_{n} \delta_{m n}
$$

with $\vartheta_{3}(\theta, q)=\sum_{n=-\infty}^{\infty} q^{n^{2} / 2} e^{i n \theta}$. That is $\vartheta_{3}$ is one of the Jacobi's theta functions. In order to use the obtained results, it is useful to compare them against those, known in literature already, e.g. see [54]. Our Eq.(70a) is in agreement with (5) of [54] if we make identifications: $\kappa=\pi$ and $c=\sqrt{2} \kappa$, where $\kappa$ is the parameter introduced in this reference. With help of such an identification we can proceed with comparison. For this purpose, following [54] we introduce yet another generating function

$$
S_{n}(t ; q):=\sum_{k=0}^{n}\left[\begin{array}{l}
n \\
k
\end{array}\right]_{q} q^{k^{2}} t^{k}
$$

so that the S-W polynomials can be written now as

$\tilde{\mathcal{S}}_{n}(t ; q)=(-1)^{n} q^{\frac{n}{2}}\left(\sqrt{(q, q)_{n}}\right)^{-1} P_{n}\left(t ; \frac{1}{2}\right) \equiv(-1)^{n} q^{\frac{2 n+1}{}}\left(\sqrt{(q, q)_{n}}\right)^{-1} S_{n}\left(-q^{\frac{1}{2}} t ; q\right)$, 
in accord with Ref.[55], page 197, provided that $0<q<1$. Comparison between generating functions (32) and (72) allows us to write as well

$$
S_{n}\left(t ; q^{-1}\right)=H_{n}\left(t q^{-n} ; q\right) \text {, or, equivalently, } H_{n}\left(t ; q^{-1}\right)=S_{n}\left(q^{-n} t ; q\right) .
$$

Using this result we can rewrite the recursion relation (33) for $H_{n}(t ; q)$ in terms of the recursion relation for $S_{n}(t ; q)$ if needed and then to repeat all the arguments with creation and annihilation operators, etc. For the sake of space, we leave this option as an exercise for our readers. Instead, to finish our discussion we would like to show how the obtained polynomials reduce to the usual Hermite polynomials in the limit $q \rightarrow 1^{-}$. For this purpose we would like to demonstrate that the recursion relation (33) is actually the recursion relation for the continuous $q$-Hermite polynomials $[56,57]$. This means that we have to demonstrate that under some conditions (to be specified) the recursion (33) is equivalent to

$$
2 x H_{n}(x \mid q)=H_{n+1}(x \mid q)+\left(1-q^{n}\right) H_{n-1}(x \mid q) .
$$

known for q-Hermite polynomials. To demonstrate the equivalence, we assume that $x=\cos \theta$ in (74) and then, let $z=e^{i \theta}$. Furthermore, we assume that

$$
H_{n}(x \mid q)=z^{n} H_{n}\left(z^{-2} ; q\right)
$$

allowing us to obtain

$$
\left(z+z^{-1}\right) z^{n} H_{n}=z^{n+1} H_{n+1}+\left(1-q^{n}\right) z^{n-1} H_{n-1} .
$$

Finally, we set $z^{-1}=\sqrt{t}$ which brings us back to Eq.(33). This time, however, we can use results known in literature for $q$-Hermite polynomials [41-43] in order to obtain at once

$$
\lim _{q \rightarrow 1^{-}} \frac{H_{n}\left(x \sqrt{\frac{1-q}{2}} \mid q\right)}{\sqrt{\frac{1-q}{2}}}=H_{n}(x),
$$

where $H_{n}(x)$ are the standard Hermitian polynomials. In view of Eq.s(72),(73), not surprisingly, the S-W polynomials are also reducible to $H_{n}(x)$. Details can be found in the same references.

\subsection{ASEP, q-deformed harmonic oscillator and spin chains}

In this subsection we would like to connect the results obtained thus far with that for XXX and XXZ spin chains. Although a connection with XXX spin chain was established already at the beginning of this section, it is extremely helpful to arrive at the same conclusions using alternative (physically inspired) arguments and methods. To understand the logic of our arguments we encourage our readers to read Appendix at this point. In it we provide a self contained summary of results related to the asymmetric simple exclusion process (ASEP), 
especially emphasizing its connection with static and dynamic properties of XXX and XXZ spin chains.

ASEP was discussed in high energy physics literature, e.g. see Ref.[58], in connection with the random matrix ensembles. To avoid repeats, we would like to use the results of Appendix in order to consider a steady-state regime only. To be in accord with literature on ASEP, we complicate matters by imposing some nontrivial boundary conditions.

In the steady -state regime Eq.(A.12) of Appendix acquires the form : $S C=$ $\Lambda$. Explicitly,

$$
S C=p_{L} E D-p_{R} D E .
$$

In the steady-state regime, the operator $S$ becomes an arbitrary c-number [59]. In view of this, following Sasamoto [60] we rewrite (78) as

$$
p_{R} D E-p_{L} E D=\zeta(D+E) .
$$

Such operator equation should be supplemented by the boundary conditions which are chosen to be as

$$
\alpha<W|E=\zeta<W| \text { and } \beta D|V>=\zeta| V>.
$$

The normalized steady-state probability for some configuration $\mathcal{C}$ can be written now as

$$
P(\mathcal{C})=\frac{<W\left|X_{1} X_{2} \cdots X_{N}\right| V>}{<W\left|C^{N}\right| V>}
$$

with the operator $X_{i}$ being either $D$ or $E$ depending on wether the $i$-th site is occupied or empty. To calculate $P(\mathcal{C})$ we need to determine $\zeta$ while assuming parameters $\alpha$ and $\beta$ to be assigned. In Appendix we demonstrate that it is possible to equate $\zeta$ to one so that, in agreement with Ref.[61], we obtain the following representation for $D$ and $E$ operators:

$$
D=\frac{1}{1-q}+\frac{1}{\sqrt{1-q}} a, \quad E=\frac{1}{1-q}+\frac{1}{\sqrt{1-q}} a^{+}
$$

converting equation (78) into (52d). In view of this mapping into $q$-deformed oscillator algebra, we can expand both vectors $|V\rangle$ and $\langle W|$ into Fourier series, e.g. $\left|V>=\sum_{m} \Omega_{m}(V)\right| m>$ where, using (57), we put $\mid m>=\Psi_{m}$. By combining equations (57) and (80) and results of Appendix we obtain the following recurrence equation for $\Omega_{n}$ :

$$
\Omega_{n}(V)\left(\frac{1-q}{\beta}-1\right)=\Omega_{n+1}(V) \sqrt{1-q^{n+1}} .
$$

Following [47], we assume that $\langle 0 \mid V\rangle=1$. Then, the above recurrence produces

$$
\Omega_{n}(V)=\frac{v^{n}}{\sqrt{(q, q)_{n}}}
$$


with parameter $v=\frac{1-q}{\beta}-1$. Analogously, we obtain:

$$
\Omega_{n}(W)=\frac{w^{n}}{\sqrt{(q, q)_{n}}}
$$

with $w=\frac{1-q}{\alpha}-1$. Obtained results exhibit apparently singular behavior for $q \rightarrow 1^{-}$. These singularities are only apparent since they cancel out when one computes quantities of physical interest discussed in both [62] and [63]. As results of Appendix indicate, such a crossover is also nontrivial physically since it involves careful treatment of the transition from XXZ to XXX antiferromagnetic spin chains. Hence, the results obtained thus far enable us to connect the partition function (27) (or (32)) with either XXX or XXZ spin chains but are not yet sufficient for making an unambiguous choice between these two models. This task is accomplished below, in the rest of this section.

\subsection{Crossover between the XXZ and XXX spin chains: connections with the KPZ and EW equations and the lattice Liouville model}

Following Derrida and Malick [63], we notice that ASEP is the lattice version of the famous Kradar-Parisi-Zhang (KPZ) equation [64]. The transition $q \rightarrow 1^{-}$ corresponds to a transition (in the sense of renormalization group analysis) from the regime of ballistic deposition whose growth is described by the KPZ equation to another regime described by the Edwards-Wilkinson (EW) equation. In the context of ASEP (that is microscopically) such a transition is discussed in detail in [65]. Alternative treatment is given in [63]. The task of obtaining the KPZ or EW equations from those describing the ASEP is nontrivial and was accomplished only very recently $[66,67]$. It is essential for us that in doing so the rules of constructing the restricted solid-on -solid (RSOS) models were used. From the work by Huse [68] it is known that such models can be found in four thermodynamic regimes. The crossover from the regime III to regime IV is described by the critical exponents of the Friedan, Qui and Shenker unitary CFT series [69]. The crossover from the regime III to regime IV happens to be relevant to the crossover from KPZ to EW regime as we would like to explain now.

As results of our Appendix indicate, the truly asymmetric simple exclusion process is associated with the XXZ model at the microscopic level and with the KPZ equation/model at the macroscopic level. Accordingly, the symmetric exclusion process is associated with the XXX model at the microscopic level and with the EW equation/model at the macroscopic level. At the level of the Bethe ansatz for open XXZ chain with boundaries full details of the crossover from the KPZ to EW regime were exhaustively worked out only very recently [70]. For the purposes of this work it is important to notice that for certain values of parameters the Hamiltonian of open XXZ spin chain model 15 with

${ }^{15}$ That is Eq.(1.3) of [70]. 
boundaries can be brought to the following canonical form

$H_{X X Z}=\frac{1}{2}\left[\sum_{j=1}^{N-1}\left(\sigma_{j}^{x} \sigma_{j+1}^{x}+\sigma_{j}^{y} \sigma_{j+1}^{y}+\frac{1}{2}\left(q+q^{-1}\right) \sigma_{j}^{z} \sigma_{j+1}^{z}\right)+\frac{1}{2}\left(q-q^{-1}\right)\left(\sigma_{1}^{z}-\sigma_{N}^{z}\right)\right]$.

In the case of ASEP we put $q=\sqrt{p_{R} / p_{L}}$ so that for physical reasons parameter $q$ is not complex. However, mathematically, we can allow $q$ to become complex. In particular, following Pasquer and Saleur [71], we can let $q=e^{i \gamma}$ with $\gamma=\frac{\pi}{\mu+1}$. For such values of $q$ use of finite scaling analysis applied to the spectrum of the above defined Hamiltonian produces the central charge

$$
c=1-\frac{6}{\mu(\mu+1)}, \mu=2,3, \ldots
$$

of the unitary CFT series. Furthermore, if $e_{i}$ is generator of the Temperley-Lieb algebra 16 , then $H_{X X Z}$ can be rewritten as [72]

$$
H_{X X Z}=-\sum_{j=1}^{N-1}\left[e_{i}-\frac{1}{4}\left(q+q^{-1}\right)\right]
$$

This fact allows us to make immediate connections with quantum groups and theory of knots and links. Below, in Section 5 we shall discuss briefly different arguments leading to analogous conclusions.

The results just described allow us to connect CFT with the exactly integrable lattice models. In view of this, one can pose the following question: Given the connection we just described, can we write down explicitly the corresponding path integral string-theoretic models reproducing results of exactly integrable lattice models at and away from criticality? Before providing the answer in the following subsection, we would like to conclude this subsection with a partial answer. In particular, we would like to mention the work by Faddeev and Tirkkonen [73] connecting the lattice Liouville model with the spin 1/2 XXZ chain. Based on this result, it should be clear that in the region $c \leq 1$ it is indeed possible by using the combinatorial analysis described above to make a link between the continuum and discrete Liouville theories 17 . It can be made in such a way that, at least at crtiticality, the results of exactly integrable 2 dimensional models are in agreement with those which are obtainable fieldtheoretically. The domain $c>1$ is physically meaningless because the models (other than string-theoretic) we discussed in this section loose their physical meaning in this region. This conclusion will be further reinforced in the next subsection.

\subsection{ASEP, vicious random walkers and string models}

We have discussed at length the role of vicious random walkers in our treatment of the Kontsevich-Witten (K-W) model [74]. Forrester [75] noticed that

\footnotetext{
${ }^{16}$ That is $e_{i}^{2}=e_{i}, e_{i} e_{i+1} e_{i}=q^{-1} e_{i}$ and $e_{i} e_{j}=e_{j} e_{i}$ for $|i-j| \geq 2$.

${ }^{17}$ The matrix $c=1$ theories will be discussed separately below.
} 
the random turns vicious walkers model is just a special case of ASEP. Further details on connections between the ASEP, vicious walkers, KPZ and random matrix theory can be found in the paper by Sasamoto [76]. In the paper by Mukhi [77] it is emphasized that while the $\mathrm{K}-\mathrm{W}$ model is the matrix model representing $c<1$ bosonic string, the Penner matrix model with imaginary coupling constant is representing $c=1$ bosonic Euclidean string on the cylinder of (self-dual) radius $R=118$. Furthermore, Ghoshal and Vafa [79] have demonstrated that $c=1, R=1$ string is dual to the topological string on a conifold singularity. We shall briefly discuss this connection below. Before doing so, it is instructive to discuss the crossover from $c=1$ to $c<1$ string models in terms of vicious walkers. To do so we shall use some results from our work on $\mathrm{K}-\mathrm{W}$ model and from the paper by Forrester [75].

Thus, we would like to consider a planar lattice where at the beginning we place only one directed path $P$ : from $(a, 1)$ to $(b, N) \sqrt{19}$. The information about this path can be encoded into multiset $\operatorname{Hor}_{y}(P)$ of $y$-coordinates of the horizontal steps of $P$. Let now

$$
w(P)=\prod_{i=\operatorname{Hor}_{y}(P)} x_{i}
$$

Using these definitions, the extension of these results to an assembly of directed random vicious walkers is given as a product: $W(\hat{P}) \equiv w\left(P_{1}\right) \cdots w\left(P_{k}\right)$. Finally, the generating function for an assembly of such walkers is given by

$$
h_{b-a}\left(x_{1}, \ldots, x_{N}\right)=\sum_{\hat{P}} W(\hat{P}),
$$

where $W(\hat{P})$ is made of monomials of the type $x_{1}^{m_{1}} x_{2}^{m_{2}} \cdots x_{N}^{m_{N}}$ provided that $m_{1}+\cdots+m_{N}=b-a$. The following theorem discussed in our work, $\operatorname{Ref}[\mathbf{6 0}]$, is of central importance for calculation of such defined generating function.

Given integers $0<a_{1}<\cdots<a_{k}$ and $0<b_{1}<\cdots<b_{k}$, let $\mathbf{M}_{i, j}$ be the $k \times k$ matrix $\mathbf{M}_{i, j}=h_{b_{j}-a_{i}}\left(x_{1}, \ldots, x_{N}\right)$ then,

$$
\operatorname{det} \mathbf{M}=\sum_{\hat{P}} W(\hat{P}),
$$

where the sum is taken over all sequences $\left(P_{1}, \ldots, P_{k}\right) \equiv \hat{P}$ of nonintersecting lattice paths $P_{i}:\left(a_{i}, 1\right) \rightarrow\left(b_{i}, N\right), i=1-k$.

Let now $a_{i}=i$ and $b_{j}=\lambda_{i}+j$ so that $1 \leq i, j \leq k$ with $\lambda$ being a partition of $N$ with $k$ parts then, $\operatorname{det} \mathbf{M}=s_{\lambda}\left(x_{1}, \ldots, x_{N}\right)$, where $s_{\lambda}(\mathbf{x})$ is the Schur polynomial. In our work [74] we demonstrated that in the limit $N \rightarrow \infty$ such defined Schur polynomial coincides with the partition (generating) function for the Kontsevich model. To obtain results of Forrester requires us to apply some additional efforts. These are worth discussing.

\footnotetext{
${ }^{18}$ This was initially demonstrated by Distler and Vafa [78].

${ }^{19}$ Very much in the same way as we have discussed in Section 2.
} 
Unlike the $\mathrm{K}-\mathrm{W}$ case, this time, we need to discuss the continuous random walks in the plane. Let $x$-coordinate represent "space" while $y$-coordinate"time". If initially $(t=0)$ we had $k$-walkers in positions $-L<x_{1}<x_{2}<$ $\cdots<x_{k}<L$, the same order should persist $\forall t>0$. At each tick of the clock each walker is moving either to the right or to the left with equal probability $p$ (that is we are in the regime appropriate for the XXX spin chain in the ASEP terminology). As before, let $\mathbf{x}_{0}=\left(x_{1,0}, \ldots, x_{k, 0}\right)$ be the initial configuration of $k$-walkers and $\mathbf{x}_{f}=\left(x_{1, f}, \ldots, x_{k, f}\right)$ be the final configuration at time $t$. To calculate the total number of walks starting at time $t=0$ at $\mathbf{x}_{0}$ and ending at time $t$ at $\mathbf{x}_{f}$ we need to know the probability distribution $W_{k}\left(\mathbf{x}_{0} \rightarrow \mathbf{x}_{f} ; t\right)$ that the walkers proceed without bumping into each other. Should these random walks be totally uncorrelated, we would obtain for the probability distribution the standard Gaussian result

$$
W_{k}^{0}\left(\mathbf{x}_{0} \rightarrow \mathbf{x}_{f} ; t\right)=\frac{\exp \left\{-\left(\mathbf{x}_{f}-\mathbf{x}_{0}\right)^{2} / 2 D t\right\}}{(2 \pi D t)^{k / 2}} .
$$

In the present case the walks are restricted (correlated) so that the probability should be modified. This modification can be found in the work by Fisher and Huse [80]. These authors obtain

$$
W_{k}\left(\mathbf{x}_{0} \rightarrow \mathbf{x}_{f} ; t\right)=U_{k}\left(\mathbf{x}_{0}, \mathbf{x}_{f} ; t\right) \frac{\exp \left\{-\left(\mathbf{x}_{f}^{2}+\mathbf{x}_{0}^{2}\right) / 2 D t\right\}}{(2 \pi D t)^{k / 2}}
$$

with

$$
U_{k}\left(\mathbf{x}_{0}, \mathbf{x}_{f} ; t\right)=\sum_{g \in S_{k}} \varepsilon(g) \exp \left[\frac{\left(\mathbf{x}_{f} \cdot g \mathbf{x}_{0}\right)}{D t}\right] .
$$

In this expression $\varepsilon(g)= \pm 1$, and the index $g$ runs over all members of the symmetric group $S_{k}$. Mathematically, following Gaudin [81], this problem can be looked upon as a problem of a random walk inside the $k$-dimensional kaleidoscope (Weyl cone) usually complicated by imposition of some boundary conditions on walks at the walls of the cone. Connection of such random walk problem with random matrices was discussed by Grabiner [82] whose results were very recently improved and generalized by Krattenthaller [83]. In the work by de Haro some applications of Grabiner's results to high energy physics were considered [84]. Here we would like to approach the same class of problems based on the results obtained in this paper. In particular, some calculations made in [80] indicate that for $L \rightarrow \infty$ with accuracy up to $O\left(L^{2} / D t\right)$ it is possible to rewrite $U_{k}\left(\mathbf{x}_{0}, \mathbf{x}_{f} ; t\right)$ as follows:

$$
U_{k}\left(\mathbf{x}_{0}, \mathbf{x}_{f} ; t\right) \simeq \operatorname{const} \Delta\left(\mathbf{x}_{f}\right) \Delta\left(\mathbf{x}_{0}\right) /(D t)^{n_{k}}+O\left(L^{2} / D t\right)
$$

with $n_{k}=(1 / 2) k(k-1)$ and const $=1 / 1 ! 2 ! \cdots(k-1) !$ and $\Delta(\mathbf{x})$ being the Vandermonde determinant, i.e.

$$
\Delta(\mathbf{x})=\prod_{i<j}\left(x_{i}-x_{j}\right) .
$$


Next, from standard texts in probability theory it is known that non-normalized expression for $W_{k}^{0}\left(\mathbf{x}_{0} \rightarrow \mathbf{x}_{f} ; t\right)$ in the limit of long times provides the number of random walks of $n$ steps (since $n \rightleftarrows t$ ) from point $\mathbf{x}_{0}$ to point $\mathbf{x}_{f}$. Hence, the same must be true for $W_{k}\left(\mathbf{x}_{0} \rightarrow \mathbf{x}_{f} ; t\right)$ and, therefore, $W_{k}\left(\mathbf{x}_{0} \rightarrow \mathbf{x}_{f} ; t\right) \approx \operatorname{det} \mathbf{M}$. Consider such walks for which $\mathbf{x}(t=0) \equiv \mathbf{x}_{0}=\mathbf{x}\left(t=t_{f}\right) \equiv \mathbf{x}_{f}$. Then, using (90) and (92) we obtain the probability distribution for the Gaussian unitary ensemble [85], i.e.

$$
W_{k}\left(\mathbf{x}_{0}=\mathbf{x}_{f} ; t\right)=\text { const } \Delta^{2}(\mathbf{x}) \exp \left(-\mathbf{x}^{2}\right) .
$$

Some additional manipulations (described in our work [74]) using this ensemble lead directly to the K-W matrix model. Forrester [75] considered a related quantity- the probability that all $k$ vicious walkers will survive at time $t_{f}$. To obtain this probability requires integration of $W_{k}\left(\mathbf{x}_{0} \rightarrow \mathbf{x}_{f} ; t\right)$ over the simplex $\Delta$ defined by $-L<x_{1}<x_{2}<\cdots<x_{k}<L 20$. Without loss of generality, it is permissible to use $W_{k}\left(\mathbf{x}_{0}=\mathbf{x}_{f} ; t\right)$ instead of $W_{k}\left(\mathbf{x}_{0} \rightarrow \mathbf{x}_{f} ; t\right)$ in calculating such a probability. Then, the obtained result coincides (up to a constant) with the partition function for topological gravity $\mathcal{Z}(g)$, Eq.(3.1) of [86,8721). Furthermore, such defined partition function can be employed to reproduce back the Hermite polynomial $H_{k}(x)$ defined by (77) which has an interpretation as the wavefunction(amplitude) of the FZZT $D$-brane $[86,87]$. Specifically, we have

$$
\begin{aligned}
& <\operatorname{det}(x-M)>=\left(\frac{g}{4}\right)^{\frac{n}{2}} H_{k}\left(x \sqrt{\frac{1}{g}}\right) \\
& =\frac{1}{\mathcal{Z}(g)} \int d M \operatorname{det}(x-M) e^{-\frac{1}{g} \operatorname{tr} M^{2}} .
\end{aligned}
$$

This expression is a special case of Heine's formula representing monic orthogonal polynomials through random matrices. In the above formula $k$ is related to the size of Hermitian matrix $M$ and $g$ is the coupling constant.

Following Forrester [75], the result, Eq.(90), can be treated more accurately (albeit a bit speculatively) if, in addition to the parameter $D$ we introduce another parameter $a$ - the spacing between random walkers at time $t=0$. Furthermore, if the time direction is treated as space direction (as it is commonly done for $1 \mathrm{~d}$ quantum systems in connection with $2 \mathrm{~d}$ classical systems), then yet another parameter $\tau(k, t)$ should be introduced which effectively renormalizes $D$. This eventually causes us to replace $\mathcal{Z}(g)$ by the following integral (up to a constant)

$$
\hat{\mathcal{Z}}(g)=\prod_{i=1}^{k} \int_{-\infty}^{\infty} d x_{i} \exp \left(-\frac{1}{2 g} \ln ^{2} x_{i}\right) \prod_{i<j}\left(x_{i}-x_{j}\right)^{2} \equiv \int d M e^{-\frac{1}{2 g} \operatorname{tr}(\ln M)^{2}} .
$$

${ }^{20}$ Such type of integration is described in detail in our papers, Parts I and II, from which it follows that in the limit $L \rightarrow \infty$ such a simplex integration can be replaced by the usual integration, i.e $\int_{\Delta} \prod_{i}^{k} d x_{i} \ldots \rightleftarrows \frac{1}{k !} \int_{-\infty}^{\infty} \prod_{i}^{k} d x_{i} \ldots$ in accord with Forrester.

${ }^{21}$ Since the hermitian matrix model given by Eq.(3.1) is just a partition function for the Gaussian unitary ensemble [85]. 
Tierz demonstrated [88] that $\hat{\mathcal{Z}}(g)$ (up to a constant) is partition function of the Chern-Simons (C-S) field theory with gauge group $U(k)$ living on 3-sphere $S^{3}$. Okuyama [87] used (95) in order to get analogous result for a D-brane amplitude in the C-S model. Using Heine's formula, he obtained the StieltjesWiegert (S-W) polynomial, our Eq.(71), which can be expressed via RogersSzego polynomial according to (73) and, hence, via the $q$-Hermite polynomial in view of the relation (75). Since in the limit $q \rightarrow 1^{-}$the $q-$ Hermite polynomial is reducible to the usual Hermite polynomial according to (77), there should be analogous procedure in going from the partition function $\hat{\mathcal{Z}}(g)$ to $\mathcal{Z}(g)$. Such a procedure can be developed, in principle, by reversing arguments of Forrester. However, these arguments are much less rigorous and physically transparent than those used in previous subsection where we discussed the crossover from XXZ to XXX model. In view of the results presented below, in the following section, we leave the problem of crossover between the matrix ensembles outside the scope of this paper. To avoid duplications, we refer our readers to the paper by Okuyama [87] where details are provided relating our results to the topological A and B -branes.

\section{Discussion. Gaudin model as linkage between the WZNW model and K-Z equations}

We would like to remind to our readers that all results obtained thus far can be traced back to our Eq.(32) defining the Rogers-Szego polynomial which physically was interpreted as partition function for the ferromagnetic P-F spin chain 22. In previous section numerous attempts were made to connect this partition function to various known string models, even though already in Section 4.1 we came to the conclusion that in the limit of infinitely long chains the antiferromagnetic version of P-F spin chain can be replaced by the spin $1 / 2$ antiferromagnetic XXX chain. If this is so, then from literature it is known that the behavior of such spin chain is described by the $S U_{1}(2)$ WZNW model [44]. Hence, at the physical level of rigor the problem of connecting Veneziano amplitudes to physical models can be considered as completely solved. Here we argue that at the mathematical level of rigor this is not quite so yet. This conclusion concerns not only problems discussed in this paper but, in general, the connection between WZNW models, spin chains and K-Z equations.

It is true that the K-Z equations and WZNW model are inseparable from each other [44] but the extent to which spin chains can be directly linked to both the WZNW models and K-Z equations remains to be explained. Following Varchenko [89], we notice that the link between the K-Z equations and WZNW models can be made only with help of the Gaudin model, while the connection with spin chains can be made only by using the quantum version of the K-

\footnotetext{
${ }^{22}$ The antiferromagnetic version of P-F spin chain is easily obtainable from this ferromagnetic version as discussed in Section 3.
} 
$\mathrm{Z}$ equation. Such quantized version of the $\mathrm{K}-\mathrm{Z}$ equation is not immediately connected with the standard WZNW model as discussed in many places [89, 90]. For the sake of space we refer our readers to these references and to our latest work connecting Veneziano amplitudes with Gaudin models [27] in which we also discuss in detail physical implications of such a connection.

\section{Appendix. Basics of ASEP}

\section{A.1. Equations of motion and spin chains}

The one dimensional asymmetric simple exclusion process (ASEP) had been studied for some time [91]. The purpose of this Appendix is to summarize the key features of this process which are of immediate relevance to the content of this paper. To this purpose, following Schütz [92], we shall briefly describe the ASEP with sequential updating. Let $B_{N}:=\left\{x_{1}, \ldots, x_{N}\right\}$ be the set of sites of one dimensional lattice arranged at time $t$ in such a way that $x_{1}<x_{2}<\cdots<x_{N}$. It is expected that each time update will not destroy this order.

Consider first the simplest case of $N=1$. Let $p_{R}\left(p_{L}\right)$ be the probability of a particle located at the site $x$ to move to the right (left) then, after transition to continuous time, the master equation for the probability $P(x ; t)$ can be written as follows

$$
\frac{\partial}{\partial t} P(x ; t)=p_{R} P(x-1 ; t)+p_{L} P(x+1 ; t)-P(x ; t) .
$$

Assuming that $P(x ; t)=\exp (-\varepsilon t) P(x)$ so that that

$$
P(x ; t)=\int_{0}^{2 \pi} d p \exp (-\varepsilon t) f(p) \exp (i p x), p \in[0,2 \pi), \text { we obtain the dispersion }
$$

relation for the energy $\varepsilon(p)$ :

$$
\varepsilon(p)=p_{R}\left(1-e^{-i p}\right)+p_{L}\left(1-e^{i p}\right) .
$$

The initial condition $P(x ; 0)=\delta_{x, y}$ determines the amplitude $f(p)=e^{-i p y} / 2 \pi$ and finally yields

$$
P(x ; t \text { । } y ; 0)=\frac{1}{2 \pi} \int_{0}^{2 \pi} d p e^{-\varepsilon(p) t} e^{-i p y} e^{i p x}=e^{-\left(q+q^{-1}\right) D t} q^{x-y} I_{x-y}(2 D t),
$$

where $q=\sqrt{p_{R} / p_{L}}, D=\sqrt{p_{R} p_{L}}$ and $I_{n}(2 D t)$ is the modified Bessel function. These results can be easily extended to the case $N=2$. Indeed, for this case we obtain the following equation of motion

$$
\begin{aligned}
\varepsilon P\left(x_{1}, x_{2}\right)= & -p_{R}\left(P\left(x_{1}-1, x_{2}\right)+P\left(x_{1}, x_{2}-1\right)-2 P\left(x_{1}, x_{2}\right)\right) \\
& -p_{L}\left(P\left(x_{1}+1, x_{2}\right)+P\left(x_{1}, x_{2}+1\right)-2 P\left(x_{1}, x_{2}\right)\right)
\end{aligned}
$$


which should be supplemented by the boundary condition

$$
P(x, x+1)=p_{R} P(x, x)+p_{L} P(x+1, x+1) \forall x .
$$

Imposition of this boundary condition allows us to look for a solution of (A.4) in the (Bethe ansatz) form

$$
P\left(x_{1}, x_{2}\right)=A_{12} e^{i p_{1} x_{1}} e^{i p_{2} x_{2}}+A_{21} e^{i p_{2} x_{1}} e^{i p_{1} x_{2}}
$$

yielding $\varepsilon\left(p_{1}, p_{2}\right)=\varepsilon\left(p_{1}\right)+\varepsilon\left(p_{2}\right)$. Use of the boundary condition (A.5) fixes the ratio (the S-matrix) $S_{12}=A_{12} / A_{21}$ as follows:

$$
S\left(p_{1}, p_{2}\right)=-\frac{p_{R}+p_{L} e^{i p_{1}+i p_{2}}-e^{i p_{1}}}{p_{R}+p_{L} e^{i p_{1}+i p_{2}}-e^{i p_{2}}} .
$$

To connect this result with the quantum spin chains, we consider the case of symmetric hopping first. In this case we have $p_{R}=p_{L}=1 / 2$ so that (A.7) is reduced to

$$
S_{X X X}\left(p_{1}, p_{2}\right)=-\frac{1+e^{i p_{1}+i p_{2}}-2 e^{i p_{1}}}{1+e^{i p_{1}+i p_{2}}-2 e^{i p_{2}}}
$$

from this result we can recognize the $S$ matrix for the XXX spin 1/2 Heisenberg ferromagnet [81]. If $p_{R} \neq p_{L}$, to bring (A.7) in correspondence with the spin chain $S$ - matrix requires additional efforts. Following Gwa and Spohn [93] we replace the complex numbers $e^{i p_{1}}$ and $e^{i p_{2}}$ in (A.7) respectively by $\mathrm{z}_{1}$ and $\mathrm{z}_{2}$. In such a form (A.7) exactly coincides with the S-matrix obtained by Gwa and Spohn23. After this we can rescale $\mathrm{z}_{i}(i=1,2)$ as follows: $z_{i}=\sqrt{\frac{q}{p}} \tilde{z}_{i}$. Substitution of such an ansatz into (A.7) leads to the result

$$
S_{X X Z}\left(\tilde{z}_{1}, \tilde{z}_{2}\right)=-\frac{1+\tilde{z}_{1} \tilde{z}_{2}-2 \Delta \tilde{z}_{1}}{1+\tilde{z}_{1} \tilde{z}_{2}-2 \Delta \tilde{z}_{2}}
$$

provided that $2 \Delta=1 / \sqrt{p_{L} p_{R}}$. For $p_{R}=p_{L}=1 / 2$ we obtain $\Delta=1$ as required for the XXX chain 24 If, however, $p_{R} \neq p_{L}$, then, the obtained $S$ matrix coincides with that known for the XXZ model [81] if we again relabel $\tilde{z}_{i}$ by $e^{i p_{i}}$ which is always permissible since the parameter $p$ is determined by the Bethe equations (to be discussed below) anyway.

In the case of XXZ spin chain it is customary to think about the massless $-1 \leq \Delta \leq 1$ and massive $|\Delta|>1$ regime. The massless regime describes various CFT discussed in the text while the massive regime describes massive excitations away from criticality. As Gaudin had demonstrated [81], for XXZ chain it is sufficient to consider only $\Delta>0$ domain which makes XXZ model perfect for uses in ASEP. The cases $\Delta=0$ and $\Delta \rightarrow \infty$ also physically interesting: the first corresponds to the XY model and the second to the Ising model.

\footnotetext{
${ }^{23}$ E.g. see their Eq.(3.5).

${ }^{24}$ It should be noticed though that such a parametrization is not unique. For instance, following [70] it is possible to choose a slightly different parametrization, e.g. $\Delta=-\frac{1}{2}\left(q+q^{-1}\right)$, where $q=\sqrt{p_{R} / p_{L}}$.
} 
Once the S-matrix is found, the $N$ - particle solution can be easily constructed [92]. For instance, for $N=3$ we write

$$
\begin{aligned}
\Psi\left(x_{1}, x_{2}, x_{3}\right)= & \exp \left(i p_{1} x_{1}+i p_{2} x_{2}+i p_{3} x_{3}\right)+S_{21} \exp \left(i p_{2} x_{1}+i p_{1} x_{2}+i p_{3} x_{3}\right) \\
& +S_{32} S_{31} \exp \left(i p_{2} x_{1}+i p_{3} x_{2}+i p_{1} x_{3}\right)+S_{21} S_{31} S_{32} \exp \left(i p_{3} x_{1}+i p_{2} x_{2}+i p_{3} x_{3}\right) \\
& +S_{31} S_{32} \exp \left(i p_{3} x_{1}+i p_{1} x_{2}+i p_{2} x_{3}\right)+S_{32} \exp \left(i p_{1} x_{1}+i p_{3} x_{2}+i p_{2} x_{3}\right),
\end{aligned}
$$

etc. This result is used instead of $f(p)$ in (A.3) so that the full solution is given by

$$
P\left(x_{1}, \ldots, x_{N} ; t \text { । } y_{1}, \ldots, y_{N} ; 0\right)=\prod_{l=1}^{N} \frac{1}{2 \pi} \int_{0}^{2 \pi} d p_{l} e^{-\varepsilon\left(p_{l}\right) t} e^{-i p_{l} y_{l}} \Psi\left(x_{1}, \ldots, x_{N}\right) .
$$

The above picture can be refined as follows. First, the particle sitting at $x_{i}$ will move to the right(left) only if the nearby site is not occupied. Hence, the probabilities $p_{R}$ and $p_{L}$ can have values ranging from 0 to 1 . For instance, for the totally asymmetric exclusion process (TASEP) particle can move to the right with probability 1 if the neighboring site to its right is empty. Otherwise the move is rejected. Since under such circumstances particle can never move to the left, there must be a particle source located next to the leftmost particle position and the particle sink located immediately after the rightmost position. After imposition of emission and absorption rates for these sources and sinks, we end up with the Bethe ansatz complicated by the imposed boundary conditions. Although in the case of solid state physics these conditions are normally assumed to be periodic, in the present case, they should be chosen among the solutions of the Sklyanin boundary equations [59, 72]. At more intuitive level of presentation compatible with results just discussed, the Bethe ansatz for XXZ chain accounting for the boundary effects is given in the pedagogically written paper by Alcaraz et al [94].

\section{A.2. Dynamics of ASEP and operator algebra}

To make these results useful for the main text, several additional steps are needed. For this purpose we shall follow works by Sasamoto and Wadati [95] and Stinchcombe and Schütz [59]. In doing so we rederive many of their results differently.

We begin with observation that the state of one dimensional lattice containing $N$ sites can be described in terms of a string of operators $D$ and $E$, where $D$ stands for the occupied and $E$ for empty $k$-th position along $1 d$ lattice. The non normalized probability (of the type given in (A.11)) can then be presented as a sum of terms like this $\langle E E D E D D D \cdots E\rangle$ to be discussed in more detail below.

Let $C=D+E$ be the time- independent operator. Then, for the operator $D$ to be time-dependent the following commutation relations should hold

$$
S C+\dot{D} C=\Lambda,
$$




$$
\begin{gathered}
C S-C \dot{D}=\Lambda, \\
\dot{D} D+D \dot{D}=[D, S] .
\end{gathered}
$$

If $\Lambda=p_{L} C D-p_{R} D C+\left(p_{R}-p_{L}\right) D^{2} \quad\left(\right.$ or $\Lambda=p_{L} E D-p_{R} D E$ ), it is possible to determine $S$ using equations (A.12) so that we obtain,

$$
\begin{gathered}
\dot{D}=\frac{1}{2}\left[\Lambda, C^{-1}\right], \\
S=\frac{1}{2}\left\{\Lambda, C^{-1}\right\},
\end{gathered}
$$

provided that

$$
\Lambda C^{-1} D=D C^{-1} \Lambda
$$

with $\{$,$\} being an anticommutator.$

As before, we want to consider the case $p_{R}=p_{L}=\frac{1}{2}$ first. This condition leads to $\Lambda=\frac{1}{2}[C, D]$. It is convenient at this stage to introduce an operator $D_{n}$ $=C^{n-1} D C^{-n}$ and its Fourier transform $\mathcal{D}_{p}=\sum_{n} D_{n} \exp ($ ipn $)$. Using (A.13a) with $\Lambda$ just defined leads to the following equation of motion for $D_{n}$ :

$$
\dot{D}_{n}=\frac{1}{2}\left[D_{n+1}+D_{n-1}-2 D_{n}\right]
$$

to be compared with (A.1). Such a comparison produces at once $\mathcal{D}_{p}(t)=$ $\exp (-\varepsilon(p)) \mathcal{D}_{p}(0)$ so that $\varepsilon(p)=1-\cos p$ as befor 25 . Consider now Eq.(A.13c). Under conditions $p_{R}=p_{L}=\frac{1}{2}$ it can be written as

$C D C^{-1} D+D C^{-1} D C=2 D^{2}$ or, equivalently, a: 26

$$
D_{n+1} D_{n}+D_{n} D_{n-1}=2 D_{n} D_{n} .
$$

Following Gaudin [81], we consider a formal expansion $D_{n} D_{m}=\alpha \exp \left(i p_{1} n+\right.$ $\left.i p_{2} m\right)+\beta \exp \left(i p_{2} n+i p_{1} m\right)$ and use it in the previous equation in order to obtain:

$$
\begin{aligned}
& \alpha \exp \left(i p_{1}(n+1)+i p_{2} n\right)+\beta \exp \left(i p_{2}(n+1)+i p_{1} n\right) \\
& +\alpha \exp \left(i p_{1} n+i p_{2}(n-1)\right)+\beta \exp \left(i p_{2} n+i p_{1}(n-1)\right) \\
= & 2 \alpha \exp \left(i p_{1} n+i p_{2} n\right)+2 \beta \exp \left(i p_{2} n+i p_{1} n\right) .
\end{aligned}
$$

From here we also obtain:

$\left(\alpha \exp \left(i p_{1} n+i p_{2} n\right)\left(\exp \left(i p_{1}\right)+\exp \left(-i p_{2}\right)-2\right)+\beta \exp \left(i p_{1} n+i p_{2} n\right)\left(\exp \left(i p_{2}\right)+\exp \left(-i p_{1}\right)-2\right)=0\right.$ and, therefore,

$$
S\left(p_{1}, p_{2}\right) \equiv \frac{\alpha}{\beta}=-\frac{1+\exp \left(i\left(p_{1}+p_{2}\right)\right)-2 \exp \left(i p_{1}\right)}{1+\exp \left(i\left(p_{1}+p_{2}\right)\right)-2 \exp \left(i p_{2}\right)} \exp \left(i\left(p_{2}-p_{1}\right)\right)
$$

\footnotetext{
${ }^{25}$ E.g. see (A.2) with $p_{L}=p_{R}=1 / 2$

${ }^{26}$ Since using definition of $D_{n}$ we have: $D_{n+1}=C D_{n} C^{-1}$ and $D_{n-1}=C^{-1} D_{n} C$.
} 
to be compared with (A.8). An extra factor $\exp \left(i\left(p_{2}-p_{1}\right)\right)$ can be actually dropped from the $S$-matrix in view of the following chain of arguments.

Introduce the correlation function as follows

$$
\begin{aligned}
P\left(x_{1}, \ldots, x_{N} ; t \quad\right. & \left.\quad y_{1}, \ldots, y_{N} ; 0\right) \equiv Z_{N}^{-1} \operatorname{Tr}\left[D_{1}(t) \cdots D_{N}(t) C^{N}\right] \\
= & \prod_{l=1}^{N} \frac{1}{2 \pi} \int_{0}^{2 \pi} d p_{l} e^{-\varepsilon\left(p_{l}\right) t} e^{-i p_{l} y_{l}} \Psi\left(p_{1}, \ldots, p_{N}\right),
\end{aligned}
$$

where $\Psi\left(p_{1}, \ldots, p_{N}\right)=Z_{N}^{-1} \operatorname{Tr}\left[\mathcal{D}_{p_{1}}(0) \cdots \mathcal{D}_{p_{N}}(t) C^{N}\right]$ and $Z_{N}=\operatorname{tr}\left[C^{N}\right] . \quad$ In arriving at this result the definition of $\mathcal{D}_{p}(t)$, was used along with the fact that $\mathcal{C D}_{p} \mathcal{C}^{-1}=e^{-i p} \mathcal{D}_{p}$. Also, the invariance of the trace under cyclic permutations and the translational invariance of the correlation function implying that $\Psi\left(p_{1}, \ldots, p_{N}\right) \neq 0$ only if $\sum_{i} p_{i}=0$ was taken into account. These conditions are sufficient for obtaining the Bethe ansatz equations

$$
\exp \left(i p_{i} N\right)=\prod_{j=1}^{N} \tilde{S}\left(p_{i}, p_{j}\right) \forall i \neq j
$$

where $\tilde{S}\left(p_{i}, p_{j}\right)$ is the same $S$-matrix as in (A.17), except of the missing factor $\exp \left(i\left(p_{i}-p_{j}\right)\right)$ which is dropped in view of translational invariance 27 .

Extension of these results to the case $p_{R} \neq p_{L}$ is nontrivial. Because of this, we would like to provide some details not shown in the cited references. In particular, contrary to claims made in [95], we would like to demonstrate that the system of equations (A.12) obtained in [59] is equivalent to the system of equations

$$
\begin{gathered}
{[C, S]=0,} \\
C \dot{D}+C T-S D=-p_{L} C D+p_{R} D C, \\
\dot{D} C+D S-T C=p_{L} C D-p_{R} D C, \\
\dot{D} D+D \dot{D}=[T, D]
\end{gathered}
$$

obtained in [95] with the purpose of describing asymmetric processes.

To make a comparison between (A.12) and (A.20), we notice that (A.20) has operators $S$ and $T$ which cannot be trivially identified with those present in (A.12). Hence, the task lies in making such an identification. For this purpose, if we assume that $S$ in (A.20) is the same as in (A.12) then, in view of (A.20a), by subtracting (A.12b) from (A.12a) we obtain:

$$
\dot{D} C+C \dot{D}=0 .
$$

This leads to either $\dot{D}=C^{-1} \dot{D} C$ or $\dot{D}=-C \dot{D} C^{-1}$. Therefore, taking into account that, by construction, $C$ is time-independent, we obtain: $D=-C D C^{-1}+$ $\Theta$, where $\Theta$ is some diagonal time-independent matrix operator.

\footnotetext{
${ }^{27}$ Surely, in the case when the effects of boundaries should be accounted, this factor should be treated depending on the kind of boundary conditions imposed.
} 
Next, using these results we multiply (A.20b) from the right by $C^{-1}$ and (A.20c) by $C^{-1}$ from the left, and add them together in order to arrive at equation (19) of [95], i.e.

$$
2 \dot{D}=p_{R} C^{-1} D C+p_{L} C D C^{-1}-\left(p_{R}+p_{L}\right) D .
$$

Also, by multiplying this result from the right by $D$ we obtain equation (20) of [95], that is

$$
0=p_{R} D C^{-1} D C+p_{L} C D C^{-1} D-\left(p_{R}+p_{L}\right) D^{2},
$$

provided that $[T, D]=0$. That this is indeed the case can be seen from the same reference where the following result for $T$ is obtained:

$$
2 T=\left(2+p_{R}-p_{L}\right) D+p_{R} C^{-1} D C-p_{L} C D C^{-1} .
$$

Using it, we obtain: $[T, D]=0$, in view of the fact that $\left[C^{-1} D C, D\right]=0$ and $\left[C D C^{-1}, D\right]=0$ since $D=-C D C^{-1}+\Theta$ as we have already demonstrated. Furthermore, (A.24) can be straightforwardly obtained by subtracting (A.20c) (multiplied by $C^{-1}$ from the right) from (A.20a) (multiplied by $\mathrm{C}^{-1}$ from the left). Thus, contrary to the claims made in [95], equations (A.12) and (A.20) are, in fact, equivalent. Nevertheless, as claimed in [95], the system of equations (A.20) is easier to connect with the Bethe ansatz formalism.

Indeed, using already known fact that $D_{n}=C^{n-1} D C^{-n}$ Eq.(A.22) can be brought into the form:

$$
\dot{D}_{n}=\frac{1}{2}\left[p_{R} D_{n+1}+p_{L} D_{n-1}-\left(p_{R}+p_{L}\right) D_{n}\right] .
$$

This result is formally in agreement with previously obtained (A.14) for the fully symmetric case. The authors of [95] have chosen such a normalization for probabilities $p_{R}$ and $p_{L}$ that for symmetric case $p_{R}=p_{L}=1$ (instead of $p_{R}=p_{L}=1 / 2$ ). To restore the normally accepted condition $p_{R}=p_{L}=1 / 2$ requires only to rescale time appropriately. This observation is consistent with the fact that the analog of equation (A.15) (which plays the central role in Bethe ansatz-type calculations) obtained with help of (A.23) is given by

$$
p_{L} D_{n+1} D_{n}+p_{R} D_{n} D_{n-1}=\left(p_{R}+p_{L}\right) D_{n} D_{n}
$$

which holds true wether we choose $p_{R}=p_{L}=1$ or $p_{R}=p_{L}=1 / 228$. These results allow us to reobtain the $S$-matrix for the XXZ model in a way already described.

\section{A.3. Steady- state and q- algebra for the deformed harmonic os- cillator}

\footnotetext{
${ }^{28}$ It should be noted though that the authors of [95] have erroneously obtained (e.g. see their equation (23)) $p_{R} D_{n}^{2}+p_{L} D_{n+1}^{2}=\left(p_{R}+p_{L}\right) D_{n} D_{n+1}$ instead of our (A.26).
} 
Using (79) we write

$$
p_{R} D E-p_{L} E D=\zeta(D+E) .
$$

Let now $D=A_{1}+B_{1} a$ and $E=A_{2}+B_{2} a^{+}$where $A_{i}$ and $B_{i}, i=1,2$, are some $c$-numbers. Substituting these expressions back to (A.27) we obtain the following set of equations

$$
\zeta\left(A_{1}+A_{2}\right)-\varepsilon A_{1} A_{2}=C,
$$

where $C$ is some constant to be determined below, and

$$
\begin{aligned}
& \zeta B_{1}=\varepsilon B_{1} A_{2}, \\
& \zeta B_{2}=\varepsilon B_{2} A_{1} .
\end{aligned}
$$

From here we obtain: $A_{1}=A_{2}=A=\zeta / \varepsilon$, with $B_{1}, B_{2}$ being yet arbitrary c-numbers and $\varepsilon=p_{R}-p_{L}$. We can determine these numbers by comparing our results with those in [61]. This allows us to select $B_{1}=B_{2}=\frac{\xi}{\sqrt{1-q}}$, $\frac{\zeta^{2}}{\varepsilon}=\frac{\xi^{2}}{1-q}=C, q=\frac{p_{L}}{p_{R}}$ so that we obtain

$$
\begin{aligned}
& D=\frac{1}{1-q}+\frac{1}{\sqrt{1-q}} a, \\
& E=\frac{1}{1-q}+\frac{1}{\sqrt{1-q}} a^{+}
\end{aligned}
$$

and, finally,

$$
a a^{+}-q a^{+} a=1
$$

in accord with (52d).

\section{References}

[1] P.Dirac, Lectures on quantum field theory, (Yeshiva University Press, NY,1996).

[2] A.Kholodenko, Heisenberg honeycombs solve Veneziano puzzle, Int.Math.Research Forum, 4 (2009) 441-509, hep-th/0608117.

[3] A.Kholodenko, Quantum signatures of Solar System dynamics, Found.Phys. (submitted), arXiv.0707.3992.

[4] A.Kholodenko, New strings for old Veneziano amplitudes I. Analytical treatment, J.Geom.Phys.55 (2005) 50-74.

[5] A.Kholodenko, New strings for old Veneziano amplitudes II. Group-theoretic treatment, J.Geom.Phys.56 (2006)1387-1432.

[6] A.Kholodenko, New strings for old Veneziano amplitudes III. Symplectic treatment, J.Geom.Phys.56 (2006) 1433-1472.

[7] A.Kholodenko, New models for Veneziano amplitudes: 
Combinatorial, symplectic and supersymmetric aspects, Int.J.Geom.Methods in Mod. Phys.2 (2005) 563-584.

[8] P.Collins, Introduction to Regge Theory and High Energy Physics, (Cambridge University Press, Cambridge, UK,1977)

[9]. D. Bardin and G. Passarino, The Standard Model in the Making, (Clarendon Press, Oxford, UK,1999).

[10] N. Dorey, A spin chain from string theory, arXiv: 0805.4387.

[11] S. Gubser, I. Klebanov, A.Polyakov, Gauge theory correlators from non-critical string theory, Phys.Lett. 428B (1998) 105-114.

[12] J. Minahan, K. Zarembo, The Bethe ansatz for $\mathcal{N}=4$ super Yang-Mills, JHEP 0303 (2003) 013.

[13] M. Kruczenski, Spin chains and string theory, PRL 93 (2004) 161602, 4 pp.

[14] A.Cootrone, L.Martucci, J.Pons, P.Talavera, Heavy hadron spectra from spin chains and strings, JHEP 05 (2007) 027.

[15] G. 't Hooft, Topology of the gauge condition and new confinement phases in non-Abelian gauge theories, Nucl.Phys 190B (1981) 455-478.

[16] Y. Nambu, Strings, monopoles and gauge fields, Phys.Rev.D10 (1974) 4262-4268.

[17] T. Suzuki, I. Yotsuyanagi, Possible evidence for Abelian dominance in quark confinement, Phys.Rev. D 42 (1990) 4257-4260.

[18] J. Stack, S. Neiman, R.Wensley, String Tension from Monopoles in SU(2) Lattice Gauge Theory, Phys Rev.D 50 (1994) 3399-3405.

[19] Y. Cho, Restricted gauge theory, Phys. Rev.D 21 (1980) 1080-1088.

[20] Y. Cho, D. Pak, Monopole condensation in SU(2) QCD, Phys.Rev.D 65 (2002) 074027.

[21] K-I. Kondo, Gauge-invariant gluon mass, infrared Abelian dominance, and stability of magnetic vacuum, Phys. Rev. D 74 (2006) 125003.

[22] K-I. Kondo, A. Ono, A. Shibata, T. Shinohara, T. Murakami, Glueball mass from quantized knot solitons and gauge-invariant gluon mass, J.Phys. A 39 (2006) 13767-13782.

[23] K-I. Kondo, Magnetic monopoles and center vortices as gauge-invariant topological defects simultaneously responsible for confinement, arXiv: 0802.3829 .

[24] L. Faddeev, Knots as possible excitations of the quantum Yang-Mills fields, arXiv: 0805.1624.

[25] D. Auckley, L. Kapitanski, J. Speight, Geometry and analysis in nonlinear sigma models, St.Petersburg Math. J. 18 (2007) 1-19.

[26] Y.Cho, D.Pak, P.Zhang, New interpretation of Skyrme theory, arXiv: hep-th/0404181

[27] A.Kholodenko, Veneziano amplitudes, spin chains and Abelian reduction of QCD, arXiv: 0810.0250

[28] A.Polychronakos, Exact spectrum of SU(n) spin chain with inverse square exchange, Nucl.Phys. B419 (1994) 553-566.

[29] R.Stanley, Combinatorics and commutative algebra 
(Birkhäuser, Boston 1996).

[30] S.Ghorpade and G.Lachaud, Hyperplane sections of Grassmannians and the number of MDS linear codes, Finite Fields \&

Their Applications 7 (2001) 468-476.

[31] R.Stanley, Enumerative combinatorics, Vol.1

(Cambridge University Press, Cambridge, U.K.1999).

[32] S.Mohanty, Lattice path counting and applications,

(Academic Press, NY, 1979).

[33] R.Bott and L.Tu, Differential forms in algebraic topology, (Springer-Verlag, Berlin, 1982).

[34] J.Schwartz, Differential geometry and topology, (Gordon and Breach, Inc., NY, 1968).

[35] M.Stone, Supersymmetry and quantum mechanics of spin, Nucl.Phys. B 314 (1989) 557-569.

[36] O.Alvarez, I Singer and P.Windey, Quantum mechanics and the geometry of the Weyl character formula,

Nucl.Phys.B 337 (1990) 467-482.

[37] A.Polyakov, Gauge fields and strings, (Harwood Academic Publ., NY, 1987).

[38] H. Frahm, Spectrum of a spin chain with invese square exchange, J.Phys.A 26 (1993) L473-479.

[39] A.Polychronakos, Generalized statistics in one dimension, hep-th/9902157.

[40] A.Polychronakos, Physics and mathematics of Calogero particles, hep-th/0607033.

[41] K.Hikami, Yangian symmetry and Virasoro character in a lattice spin system with long -range interactions,

Nucl.Phys.B 441 (1995) 530-568.

[42] E.Melzer, The many faces of a character, hep-th/9312043.

[43] R.Kedem, B.McCoy and E.Melzer, The sums of Rogers, Schur and Ramanujian and the Bose-Fermi correspondence in $1+1$ dimensional quantum field theory, hep-th/9304056.

[44] A.Tsvelik, Quantum Field Theory in Condensed Matter Physics, (Cambridge University Press, Cambridge, U.K., 2003).

[45] J.Goldman and J.C.Rota, The number of subspaces of a vector space, in Recent Progress in Combinatorics, pp.75-83. (Academic Press, NY,1969).

[46] V.Kac and P.Cheung, Quantum calculus (Springer-Verlag, Berlin , 2002).

[47] G.Andrews, The theory of partitions (Addison-Wesley Publ.Co., London,1976).

[48] D.Galetti, Realization of the q-deformed harmonic oscillator: Rogers-Szego and Stiltjes-Wiegert polynomials, Brazilian Journal of Physics 33 (2003)148-157.

[49] M.Chaichian, H.Grosse and P.Presnajer, Unitary representations of the q-oscillator algebra, J.Phys.A 27 (1994) 2045-2051. 
[50] R. Floreani and L.Vinet, Q-orthogonal polynomials and the oscillator quantum group, Lett.Math.Phys. 22 (1991) 45-54.

[51] H.Karabulut, Distributed Gaussian polynomials as q-oscillator eigenfunctions, J.Math.Phys.47 (2006) 013508.

[52] A.Macfarlane, On q-analogues of the quantum harmonic oscillator and the quantum group $S U(2)_{q}$, J.Phys.A 22 (1989) 4581-4588.

[53] H.Karabulut and E.Siebert, Distributed Gaussian polynomials and associated Gaussian quadratures, J.Math.Phys.38 (1997) 4815-4831

[54] A.Atakishiev and Sh.Nagiyev, On the Rogers-Szego polynomials, J.Phys.A 27 (1994) L611-615.

[55] G.Gasper and M.Rahman, Basic hypergeometric series, (Cambridge University Press, Cambridge, U.K, 1990).

[56] R.Koekoek and R.Swarttouw, The Askey- scheme of hypergeometric orthogonal polynomials and its q-analogs, arXiv:math/9602214.

[57] M.Ismail, Classical and Quantum Orthogonal Polynomials of One Variable (Cambridge University Press, Cambridge, U.K, 2005).

[58] T.Nagao and T.Sasamoto, Asymmetric simple exclusion process and modified random matrix ensembles, Nucl.Phys.B 699 (2004) 487-502.

[59] R.Stinchcombe and G.Schutz, Application of operator algebras to stochastic dynamics and Heisenberg chain,

PRL 75 (1995) 140-144.

[60] T.Sasamoto, One-dimensional partially asymmetric simple exclusion process with open boundaries: orthogonal polynomials approach, J.Phys.A 32 (1999) 7109-7131.

[61] R.Blythe, M.Ewans, F.Colaiori and F.Essler, Exact solution of a partially asymmetric exclusion model using a deformed oscillator algebra, J.Phys.A 33 (2000) 2313-2332.

[62] B.Derrida, M.Ewans, V.Hakim and V.Pasquer, Exact solution of a $1 \mathrm{~d}$ asymmetric exclusion model using a matrix formulation, J.Phys A 26 (1993) 1493-1517.

[63] B.Derrida and K.Malick, Exact diffusion constant for the onedimensional partially asymmetric exclusion model, J.Phys.A 30 (1997) 1031-1046.

[64] M.Kardar, G.Parisi and Yi-Ch.Zhang, Dynamic scaling of growing interfaces, PRL 56 (1986) 889-892.

[65] T.Sasamoto, S.Mori and M.Wadati, One-dimensional asymmetric exclusion model with open boundaries, J.Phys.Soc.Japan 65 (1996) 2000-2008.

[66] T. Oliviera, K.Dechoum, J.Redinz and F. Aarao Reis, Universal and nonuniversal features of the crossover from linear to nonlinear interface growth, Phys.Rev. E 74 (2006) 011604.

[67] A.Lazarides, Coarse-graining a restricted solid-on -solid model, Phys.Rev.E 73 (2006) 041605. 
[68] D.Huse, Exact exponents for infinitely many new multicritical points, Phys.Rev.B 30 (1984) 3908-3917.

[69] D.Friedan, Z.Qui and S.Shenker, Conformal invariance,unitarity and critical exponents in two dimensions, PRL 52 (1984) 1575-1580.

[70] J.de Gier and F.Essler, Exact spectral gaps of the asymmetric exclusion process with open boundaries, J.Sat.Mech.(2006) P12011.

[71] V.Pasquer and H.Saleur, Common structures between finite systems and conformal field theories through quantum groups, Nucl.Phys. B 330 (1990) 523-547.

[72] C.Gomez, m.Ruiz-Altaba and G.Sierra, Quantum Groups in Two-Dimensional Physics, (Cambridge University Press, Cambridge, UK,1996).

[73] L.Faddeev and O.Tirkkonen, Connections of the Liouville model and XXZ spin chain, Nucl.Phys.B 453 (1995) 647-669.

[74] A.Kholodenko, Kontsevich-Witten model from 2+1 gravity: New exact combinatorial solution, J.Geom.Phys.43 (2002) 45-91

[75] P.Forrester, Vicious random walkers in the limit of a large number of walkers, J.Stat.Phys. 56 (1989) 767-784.

[76] T.Sasamoto, Fluctuations of the one-dimensional asymmetric exclusion process using random matrix techniques, J.Stat.Mech. (2007) P07007.

[77] A.Mukherjee and S.Mukhi, $\mathrm{c}=1$ matrix models: equivalences and open-closed string duality, JHEP 0510 (2005) 099.

[78] J.Distler and C.Vafa, A critical matrix model at $\mathrm{c}=1$, Mod.Phys.Lett.A 6 (1991) 259-267.

[79] D.Ghoshal and C.Vafa, $\mathrm{c}=1$ strting as the topological theory on a conifold, Nucl.Phys. B 453 (1995) 121-128.

[80] D.Huse and M.Fisher, Commensurate melting, domain walls, and dislocations, Phys.Rev.B29 (1984) 239-248.

[81] M.Gaudin, La Function D'onde de Bethe, (Masson, Paris, 1983).

[82] D.Grabiner, Brownian motion in a Weyl chamber, non -colliding particles, and random matrices, arXiv: math.RT/9708207.

[83] C. Krattenhaler, Asymptotics for random walks in alcoves of affine Weyl groups, arXiv: math/0301203.

[84] S.de Haro, Chern-Simons theory, 2d Yang -Mills, and Lie algebra wanderers, Nucl.Phys. B730 (2005) 312-351.

[85] M.Mehta, Random Matrices (Elsevier, Amsterdam, 2004).

[86] J.Maldacena, G.Moore, N.Seiberg and D.Shih, Exact vs. semiclassical target space of the minimal string, JHEP 0410 (2004) 020.

[87] K.Okuyama, D-brane amplitudes in topological string on 
conifold, Phys.Lett.B 645 (2007) 275-280.

[88] M.Tierz, Soft matrix models and Chern-Simons partition functions, Mod.Phys. A 19 (2004) 1365-1378.

[89] A.Varchenko, Special Functions, KZ Type Equations, and Representation Theory

(AMS Publishers, Providence, RI, 2003).

[90] P.Etingof, I.Frenkel and A.Kirillov Jr., Lectures on

Representation Theory and Knizhnik-Zamolodchikov

Equations, (AMS Publishers, Providence, RI,1998).

[91] R. Blythe and M.Evans, Nonequilibrium steady states of matrix-product form: a solver guide,

J.Phys.A 40 (2007) R333.

[92] G.Schütz, Exact solution of the master equation for the asymmetric exclusion process, J.Stat.Phys. 88 (1997) 427-445.

[93] L.Gwa and H.Spohn, Bethe solution for the dynamical-scaling exponent of the noisy Burgers equation,

Phys.Rev.A 46 (1992) 844-854.

[94] F.Alcaraz, M.Barber, M.Batchelor, R.Baxter and G.Quispei, Surface exponents of the quantum XXZ, Ashkin-Teller and Potts models, J.Phys.A 20 (1987) 6397-6416.

[95] T.Sasamoto and M.Wadati, Dynamic matrix product ansatz and Bethe ansatz equation for asymmetric exclusion process with periodic boundary, J.Phys.Soc.Jpn. 66 (1997) 279-282. 